\title{
A Data-driven Framework for Long-Range Aircraft Conflict Detection and Resolution
}

\author{
SAMET AYHAN, University of Maryland \\ PABLO COSTAS, Boeing Research \& Technology Europe \\ HANAN SAMET, University of Maryland
}

At the present time, there is no mechanism for Air Navigation Service Providers (ANSPs) to probe new flight plans filed by the Airlines Operation Centers (AOCs) against the existing approved flight plans to see if they are likely to cause conflicts or bring sector traffic densities beyond control. In the current Air Traffic Control (ATC) operations, aircraft conflicts and sector traffic densities are resolved tactically, increasing workload and leading to potential safety risks and loss of capacity and efficiency.

We propose a novel Data-driven Framework to address a long-range aircraft conflict detection and resolution (CDR) problem. Given a set of predicted trajectories, the framework declares a conflict when a protected zone of an aircraft on its trajectory is infringed upon by another aircraft. The framework resolves the conflict by prescribing an alternative solution that is optimized by perturbing at least one of the trajectories involved in the conflict. To achieve this, the framework learns from descriptive patterns of historical trajectories and pertinent weather observations and builds a Hidden Markov Model (HMM). Using a variant of the Viterbi algorithm, the framework avoids the airspace volume in which the conflict is detected and generates a new optimal trajectory that is conflict free. The key concept upon which the framework is built is the assumption that the airspace is nothing more than a horizontally and vertically concatenated set of spatio-temporal data cubes where each cube is considered as an atomic unit. We evaluate our framework using real trajectory datasets with pertinent weather observations from two continents and demonstrate its effectiveness for strategic CDR.

\section{CCS Concepts: • Information systems $\rightarrow$ Data analytics; • Computing methodologies $\rightarrow$ Dynamic programming for Markov decision processes; • Applied computing $\rightarrow$ Aerospace;}

Additional Key Words and Phrases: Aircraft conflict detection and resolution, air traffic management, prescriptive analytics, Hidden Markov model, time series

\footnotetext{
S. Ayhan is Senior Engineer at Boeing Research \& Technology.

This work was supported in part by the NSF under Grants IIS-13-20791 and IIS-18-16889. We are grateful to the Boeing Research \& Technology Europe, a member of the project DART [56] team, for providing the European trajectory data used in this study. The project DART has received funding from the SESAR Joint Undertaking under Grant Agreement No. 699299 under the European Union's Horizon 2020 Research and Innovation Programme.

Authors' addresses: S. Ayhan and H. Samet, University of Maryland, Department of Computer Science, College Park, MD 20742; emails: \{sayhan, hjs\}@cs.umd.edu; P. Costas, Boeing Research \& Technology Europe, Av. Sur del Aeropuerto de Barajas, 38, Madrid, Spain 28042; email: pablo.costas@boeing.com.

Permission to make digital or hard copies of all or part of this work for personal or classroom use is granted without fee provided that copies are not made or distributed for profit or commercial advantage and that copies bear this notice and the full citation on the first page. Copyrights for components of this work owned by others than ACM must be honored. Abstracting with credit is permitted. To copy otherwise, or republish, to post on servers or to redistribute to lists, requires prior specific permission and/or a fee. Request permissions from permissions@acm.org. (C) 2019 Association for Computing Machinery.

2374-0353/2019/09-ART24 \$15.00

https://doi.org/10.1145/3328832
} 


\section{ACM Reference format:}

Samet Ayhan, Pablo Costas, and Hanan Samet. 2019. A Data-driven Framework for Long-Range Aircraft Conflict Detection and Resolution. ACM Trans. Spatial Algorithms Syst. 5, 4, Article 24 (September 2019), 23 pages.

https://doi.org/10.1145/3328832

\section{INTRODUCTION}

In the present Air Traffic Management (ATM) system, Airline Operations Center (AOC) personnel file a flight plan for a particular flight. The filed flight plan usually contains two-dimensional (2D) coordinates of the fixed way points and the planned initial cruise speed and cruise altitude in addition to their speed and level changes along the route. It does not include the time information for the way points and the existing information is not routinely updated by the ATM systems. Moreover, the filed flight plan is not checked against other flight plans to probe potential interference with other aircraft or identify sector traffic complexity before the aircraft departs. Airspace sector complexity and aircraft separation assurance are dealt with tactically, usually just a few minutes before the aircraft enters the sector or is likely to have a conflict with other aircraft.

It is estimated that by 2040 the USA alone can expect an increase of more than $68 \%$ in the commercial air traffic [22]. Hence, a new concept of operations is needed to accommodate this increase in volume. To meet this challenge ahead of us, new technologies and procedures for next generation ATM are being developed in the context of the Next Generation Air Transportation System (NextGen) [20] in the USA and the Single European Sky ATM Research (SESAR) [55] in Europe. The SESAR and NextGen concept of operations requires a paradigm shift from a highly structured and fragmented system that is heavily reliant on tactical decision making and with few strategic planning functions based on uncertain information, to an integrated one based on collaborative strategic management of trajectories and information sharing [59]. In the future ATM systems to be built under NextGen and SESAR, the trajectory becomes the fundamental element of new sets of operating procedures collectively referred to as Trajectory-Based Operations (TBO). The underlying idea behind TBO is the concept of a business trajectory that the airline agrees to fly and the ANSP and airports agree to facilitate, given the safe separation provision. A business trajectory is the representation of an airline's intention with respect to a given flight that will best meet the airline's business interests. The TBO concept of operations and the notion of a business trajectory will result in increased overall predictability of air traffic, reduced costs and emissions due to lowered fuel consumption and/or time, and increased capacities. Thus, the future ATM system should offer flexibility to accommodate business trajectories, while bearing the primary goal of safety in mind. Overall, the next generation ATM paradigm shift requires more safe and efficient CDR due to fact that maintaining the separation minima among the vast volume of aircraft that fly their versions of most optimal business $4 \mathrm{D}$ trajectories becomes more challenging.

Hence, we introduce a Data-driven Framework to address the long-range aircraft CDR problem. The framework performs CDR in two steps: In the first step, given a set of predicted trajectories in the form of a $4 \mathrm{D}$ joint spatio-temporal data cubes on a 3D grid network, the framework declares a conflict if one of the aircraft's predicted trajectory segment overlaps with the other's protected zone at the same time interval in the future. Obviously, the more accurate the predicted trajectories, the more accurate the detection of the conflict as the separation minima will be on the containment volume of each predicted trajectory. In the second step, the framework builds a stochastic model, the Hidden Markov Model (HMM), that learns from the historical trajectories and their correlation with the pertinent weather parameters. Using a variant of the Viterbi algorithm, the framework prescribes an optimal solution by perturbing at least one of the $4 \mathrm{D}$ trajectories involved in the conflict. 
In summary, the contributions of this article are as follows:

- We propose a cube-shaped protected zone surrounding each aircraft that can be expanded by joining the neighboring cubes horizontally and vertically, yielding a protected zone with a desired size. This idea resonates with the assumption that an airspace is nothing more than a set of concatenated spatio-temporal data cubes around a 3D grid network, where each cube is considered as an atomic unit.

- We propose a scalable Data-driven Framework to strategically address the aircraft CDR problem. Given a set of predicted trajectories, the framework declares a conflict when a protected zone of an aircraft on its trajectory is infringed upon by another aircraft at the same time interval in the future. Upon a conflict, the framework executes our conflict resolution algorithm and prescribes a solution that resolves the conflict by perturbing at least one of the $4 \mathrm{D}$ trajectories involved in the conflict.

- We conduct extensive experiments based on real trajectory and weather data from two continents and demonstrate that our framework can detect and resolve conflicts with lateral and vertical accuracies that are within the boundaries of conventionally accepted minimum separation values, set by the ANSPs. This translates to the fact that, ANSPs can now detect and resolve potential conflicts before the aircraft depart, resulting in safer and greener airspace with more efficiency and capacity, and thereby reducing the air traffic controller workload.

In our previous work [9], we presented our Aircraft Trajectory Prediction System that shares a common ground with the current framework in a way that both the previous system and the current framework attempt to find the most likely sequence of aircraft positions given a set of weather observations. However, they differ in the following respects: (i) The previous system [9] aimed at predicting aircraft trajectories without considering any potential obstacles along the route. The proposed framework detects and resolves conflicts among the aircraft. In fact, the framework we propose in this work uses predicted trajectories generated by the previous system [9] as input. (ii) The previous system [9] utilized historical trajectories and pertinent weather observations to build an HMM that was fed into the Viterbi algorithm. Although our current framework uses the same input for an HMM during the conflict resolution phase, it employs a variant of the Viterbi algorithm. Unlike the regular Viterbi algorithm, the variant generates optimal trajectories bypassing the spatio-temporal data cubes in which the conflict is detected. (iii) Unlike the previous system [9], the current framework is scalable. It can detect and resolve conflicts among multiple aircraft. Moreover, this article is an extended version of work published in Reference [6]. We extend our previous work [6] by providing the following additional content:

- We elaborate more on the ideas of TBO and business trajectory in Section 1.

- We provide a more detailed explanation as to where our framework falls in comparison to the other systems in Section 2.

- We expand Section 4.3 with an Octree data structure figure and we provide the pertinent explanation.

- We provide time complexities for Algorithm 2 and Algorithm 3, where we present CDR for multiple aircraft in Section 4.3.

- We provide 8 additional test cases in Section 5, where we evaluate CDR for multiple aircraft.

- We provide Table 3 for the additional test cases in Section 5.1.

- We provide Figure 6 with a set of boxplots showing trajectory prediction errors that can be attributed for the CDR accuracy in Section 5.2.

- We update Figure 5 and Figure 7 based on additional test cases in Section 5.2. 
Our Data-driven Framework can be used as a ground-based strategic CDR system by air traffic flow managers to resolve potential interference among large volume of aircraft and identify high density and complex sector traffic before the aircraft depart. The set of optimized resolutions should improve ATM automation and reduce the workload of air traffic controllers. The rest of the article is organized as follows. Section 2 reviews related work. Section 3 introduces preliminary concepts followed by Section 4 where our Data-driven Framework is described. Section 5 presents the results of our experiments, while Section 6 discusses the results. Concluding remarks are drawn in Section 7.

\section{RELATED WORK}

Our study involves trajectory data that have been the subject of much work in the spatial domain with an emphasis on cars along roads [53]. The focus has been on their generation (e.g., Reference [54]), queries (e.g., References [36, 38, 39, 40, 49, 51, 52]), and matching (e.g., References [26, $35,50])$. These data are collected continuously and are quite voluminous. Instead, our focus here is on the flight domain. The problem of aircraft CDR remains an active area of research in the spatial domain and in the aviation community and has attracted the attention of many researchers. An excellent survey of various CDR methods is presented in Reference [27]. In this survey, Kuchar et al. propose a taxonomy to categorize the basic functions of CDR modeling methods. The proposed taxonomy includes: dimensions of state information (lateral, vertical, or three-dimensional); method of state propagation (nominal, worst-case, or probabilistic); conflict detection threshold; conflict resolution method (prescribed, optimized, force field, or manual); maneuvering options (speed change, lateral, vertical, or combined maneuvers); and management of multiple aircraft conflicts (pairwise or global).

Conflict detection methods can be classified as nominal, worst-case, and probabilistic techniques. The nominal technique projects the current states into the future along a single trajectory without taking uncertainties into account $[18,21,61]$. The worst-case technique assumes that an aircraft will perform any of a set of maneuvers and a conflict is predicted if any of the maneuvers could cause a conflict $[11,58,62]$. The disadvantage of the worst-case technique is that it can declare a conflict as soon as there is a minimum likelihood of a conflict within the definition of the worst-case trajectory model thereby leading to false positives. The probabilistic approach offers a balance between relying on either a single trajectory model as in the nominal technique or a set of worst-case maneuvers. Instead it models uncertainties to describe potential changes in the future trajectory [13, 29-32, 41, 64, 65].

Once a conflict has been detected, the next step in the CDR process is to initiate the conflict resolution phase by determining the course of action. The conflict resolution method and the maneuvering options are two major factors in defining the course of action. Conflict resolution methods can be categorized as (a) prescribed, (b) optimized, (c) force-field, and (d) manual. The prescribed resolution method provides a fixed maneuver based on a set of predefined procedures [14]. Hence, depending on the nature of the conflict, the predefined resolution maneuver is automatically performed, minimizing the response time. However, it does not compute the optimal resolution path for the aircraft, thereby resulting in a less efficient trajectory. The optimized method provides a conflict resolution strategy with the lowest cost based on a certain cost function (separation, fuel, time, workload, etc.) [21]. In the force-field resolution method, each aircraft is treated as a charged particle and the resolution maneuvers are defined using repulsive forces between the aircraft [66]. Although it is practical when properly applied, it may require a high level of guidance on the flight deck especially when the aircraft vary their speed over a wide range. The manual resolution method allows users to generate potential resolution options and provide feedback if the option is viable $[32,64]$. 
During the resolution phase, some CDR approaches only offer a single maneuver such as speed change [16, 24] or lateral maneuver [42] or vertical maneuver [17, 21], while others offer a combination of these maneuvers [13, 25, 33]. Obviously, the more maneuvering options the CDR approach offers, the more likely an efficient solution can be provided to a conflict. Our CDR approach has some similarities with $[15,16]$ due to fact that both approaches consider the airspace as a set of cubic cells, called the grid model, declare a conflict if one of the aircraft's predicted trajectory segments overlap with the other's protected zone, and propose an optimized 4D trajectory for the conflict resolution. However, unlike our study, they attempt to address tactical CDR (short-term and medium-term) by changing speed profiles [16] or using Particle Swarm Optimization [15].

Summarizing the above literature and comparing to the proposed prescriptive analytics approach, we make the following remarks: (i) Our CDR modeling method considers dimensions of state information as 4D (latitude, longitude, altitude, and timestamp); (ii) due to input trajectories being probabilistically computed, our conflict detection method is also considered probabilistic; (iii) our conflict resolution method is optimized, and it offers a combination of lateral and vertical maneuvers; and (iv) our framework is scalable, i.e., it can address the CDR problem among multiple aircraft. Given a set of aircraft trajectories in $4 \mathrm{D}$, our framework declares a conflict if any of the aircraft's predicted trajectory segments overlaps with the other's protected zone at the same time interval in the future. In our approach, one or more cubic cells forming the airspace is considered to be a conflict detection threshold. Next, we compute and prescribe an optimized solution that is a conflict-free $4 \mathrm{D}$ trajectory. Our approach addresses the CDR problem strategically over a time horizon of several hours to compute conflict-free $4 \mathrm{D}$ trajectories for optimal flight plans and less complex sector traffic densities. In particular, the proposed data-driven approach exploits machine learning techniques to predict conflicts and prescribe an optimized solution based on constraints introduced to the framework.

\section{PRELIMINARIES}

The primary concern of the ANSPs, for example; the FAA in the USA and EUROCONTROL in Europe is to assure safety, which is quantified by the number of resolved conflicts.

Definition 3.1. A conflict is an event in which two or more aircraft come closer than a certain distance to one another.

Definition 3.2. Separation minima are encoded by lateral and vertical separation, forming a bounding volume around each aircraft, a protected zone (PZ) also known as a buffer zone or corridor in Geographic Information Systems (GIS) applications (e.g., References $[1-3,57]$ ) and can be represented using quadtree medial axis transforms [44, 45]. Currently, the minimum lateral separation for en-route airspace is $5 \mathrm{nmi}$. It is $3 \mathrm{nmi}$ inside the terminal radar approach control (TRACON) area. The minimum vertical separation is $2,000 \mathrm{ft}$ above the altitude of 29,000 ft (FL290) and 1,000 ft below FL290. Due to fact that lateral and vertical separation are specified by single distance values, the resulting $P Z$ becomes a cylinder. Each aircraft is assumed to be surrounded by a $P Z$ that moves along the aircraft. An interesting idea is to use the Hausdorff distance [35] to define the cubes.

Definition 3.3. Conflict detection is a process that evaluates the separation between any pair of aircraft, by comparing the distance between them with the separation minima. Formally, given a pair of predicted aircraft trajectories formed by a set of aircraft positions $T_{i}=\left[p_{i 1}, p_{i 2}, \ldots, p_{i m}\right]$, $T_{j}=\left[p_{j 1}, p_{j 2}, \ldots, p_{j n}\right]$ where each point $p$ is defined by its $4 \mathrm{D}$ spatio-temporal parameters (latitude, longitude, altitude, and timestamp), distance values between them $d_{i, j}$ are computed and compared with the separation minima $d_{s}$ and a conflict is declared if any of distance values is less than the separation minima $d_{i, j}<d_{s}$. 


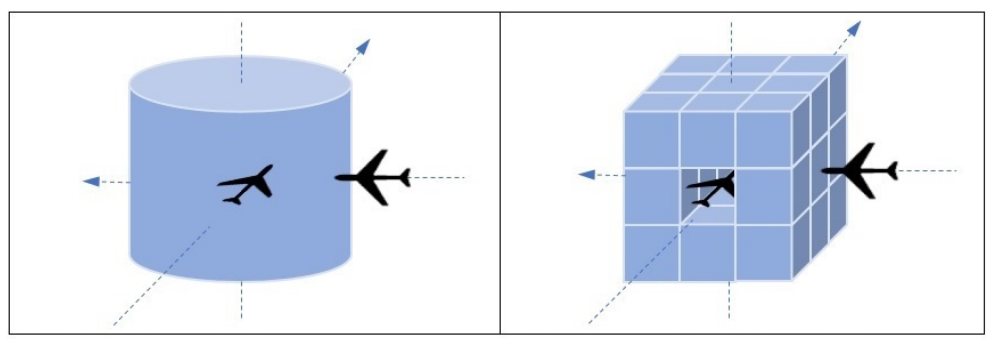

a) $P Z$ as a cylinder.

b) $P Z$ as a set of joint cubes.

Fig. 1. A sample crossing conflict and a PZ. (Left) Regular representation and (right) our unique representation.

Definition 3.4. Conflict resolution is a process that generates a feasible safe alternative trajectory by fulfilling the separation minima criteria. Formally, given a pair of predicted aircraft trajectories formed by a set of aircraft positions $T_{i}=\left[p_{i 1}, p_{i 2}, \ldots, p_{i m}\right], T_{j}=\left[p_{j 1}, p_{j 2}, \ldots, p_{j n}\right]$, upon conflict resolution, all the distance values $d_{i, j}$ between the pairs of aircraft positions are greater than the separation minima $d_{i, j}>d_{s}$.

Definition 3.5. Long-range CDR is a process in which conflict detection and resolution are carried out several hours before the potential conflict occurs. Hence, long-range CDR is strategically performed before the departure for better planning, whereas mid-range and short-range CDR are tactically performed while the aircraft is airborne.

Unlike online CDR approaches in which distance between predicted future aircraft positions are constantly computed and compared with separation minima upon receipt of each new aircraft position, our approach is offline and uses a 3D grid network as a reference system. In our approach, raw trajectories are transformed into aligned trajectories causing aircraft to move along grid points. This results in conflict queries being computed at grid points only. In addition, unlike most other CDR approaches, our framework considers a cube shaped $P Z$ surrounding each aircraft. This idea resonates with the fact that our approach creates virtual data cubes around grid points, forming an overall airspace. Each cube is defined by its centroid, the original grid point, and associated weather parameters that remain homogeneous within the cube during a period of time. With this vision, we define trajectories as a set of $4 \mathrm{D}$ joint cubes.

This uncommon representation of $4 \mathrm{D}$ trajectories enables us to view conflicts and $P Z$ from a unique perspective. Hence, in our view, $P Z$ is a cube that can be expanded by joining the neighboring cubes horizontally and vertically. The process yields a $P Z$ with a desired size. In our study, we use a $P Z$ of variable size. It can be made up of a single cube or expanded by a number of cubes in each direction on each axis reaching a larger volume. Figure 1 illustrates a $P Z$ in two different forms. The $P Z$ on the left is formed by a cylinder. The $P Z$ on the right is formed by 27 cubes. Figure 2 illustrates a sample pairwise CDR. Aircraft \#1 departs before aircraft \#2. Both aircraft move one cube at a time. In Figure 2(a). aircraft \#1 and \#2 are located at cube $J 7$ and K6, respectively. This causes a conflict as aircraft \#2 intrudes aircraft \#1's $P Z$ outlined in red. In Figure 2(b), the conflict is resolved by a lateral shift to cube $L 6$ by aircraft \#2. No conflict occurs from here on as aircraft \#1 follows cubes in gray $(K 7, L 7, M 7, N 7, O 7, P 7, R 7, S 7)$ and aircraft \#2 follows cubes in blue $(M 7, N 8, N 9, N 10, O 11, P 11, R 11, S 11, T 11)$ until they land at their pertinent airports.

\section{DATA-DRIVEN FRAMEWORK FOR CDR}

Figure 3 shows an overview of the proposed Data-driven Framework for a simplified pairwise CDR. Predicted trajectory \#1 and $\# 2$ are generated by our previous Aircraft Trajectory Prediction 


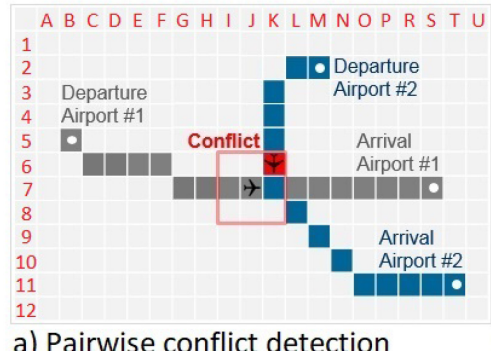

a) Pairwise conflict detection

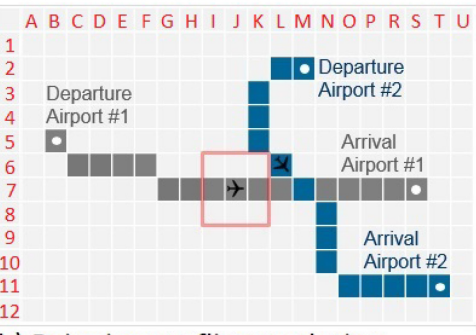

b) Pairwise conflict resolution

Fig. 2. Simplified depiction of a pairwise CDR.

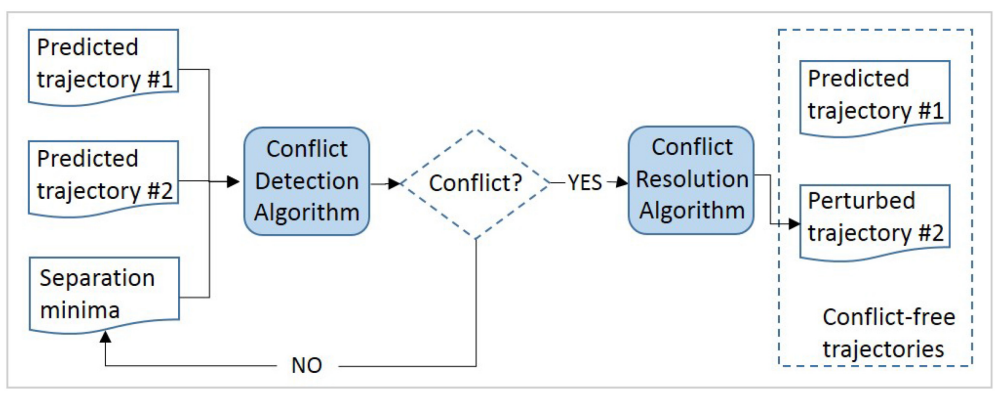

Fig. 3. Overview of our Data-driven Framework.

System [9]. Our conflict detection algorithm takes predicted trajectories along with separation minima as input. The output of the process is a data cube defined by its $4 \mathrm{D}$ position, where conflict, if any, occurs. The next process in the pipeline is the conflict resolution, where a variant of the Viterbi algorithm is performed to avoid the conflicting trajectory segment. The process perturbs at least one of the trajectories involved in the conflict, generating a new optimized path and thereby resulting in conflict-free trajectories. Separation minima may be expanded to probe what-if scenarios if the conflict detection algorithm doesn't generate a conflict.

\subsection{Pairwise Conflict Detection}

The predicted trajectories along with the size of the $P Z$ are fed into our pairwise conflict detection algorithm, presented in Algorithm 1. The algorithm declares a conflict if a $P Z$ of an aircraft is infringed upon by another aircraft at the same time interval in the future.

Formally, given a pair of predicted trajectories $T_{i}=\left[p_{i 1}, p_{i 2}, \ldots, p_{i m}\right]$ and $T_{j}=\left[p_{j 1}, p_{j 2}, \ldots, p_{j n}\right]$ where each trajectory is formed by a set of segments defined by their $4 \mathrm{D}$ spatio-temporal centroid parameters latitude, longitude, altitude, and timestamp, along with $P Z$ for trajectory $T_{i}$ in data cubes, we want to return the very first trajectory segment $p_{j s}$ if a conflict occurs and null otherwise. Note that each aircraft position along predicted trajectories are recorded once every minute. To compute this, we start with the departure point of the aircraft that departs beforehand, and keep moving forward, one grid point at a time. With the departure of the second aircraft, we compare the trajectory segment $p_{j s}$ with $P Z_{i s}$ at each time instance $t_{s}$ to see if $p_{j s}$ overlaps with $P Z_{i s}$.

Note that Algorithm 1 assumes that all the PZs have the same definition.

\subsection{Pairwise Conflict Resolution}

Our conflict resolution approach shares a common ground with our previous Trajectory Prediction System [9] as they both attempt to address an optimization problem; given a set of weather 


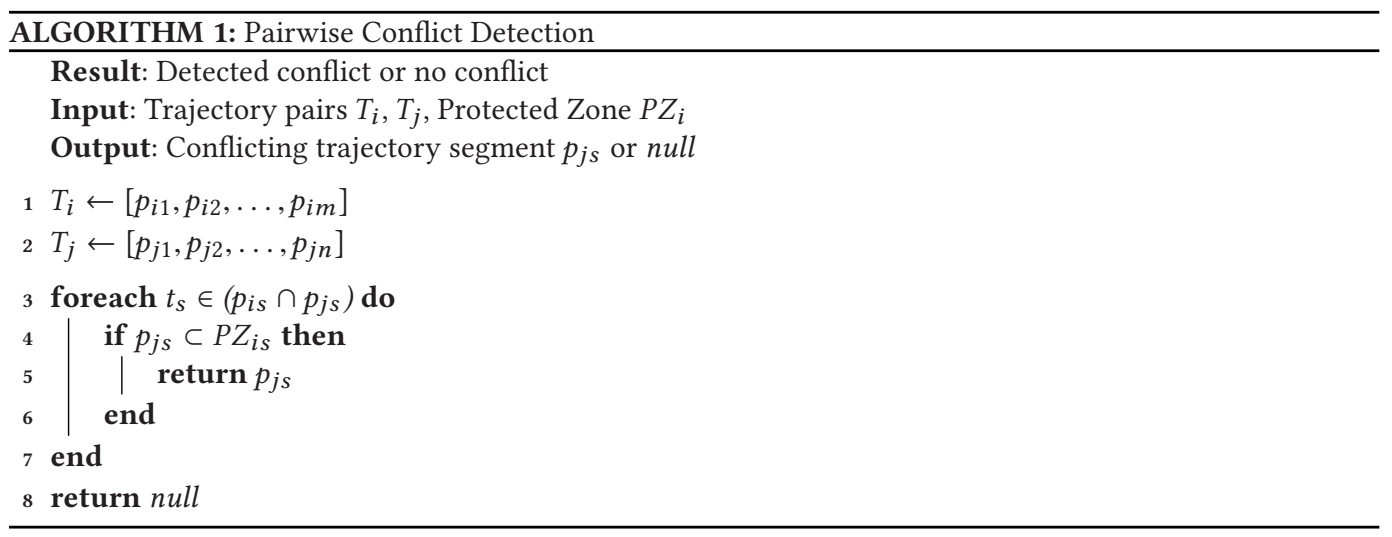

observations, what is the most likely sequence of aircraft positions? However, unlike the previous system [9], the current conflict resolution algorithm avoids the spatio-temporal data cubes where the conflict is detected. To recapitulate our approach to the optimization problem, we briefly review our previous Trajectory Prediction System [9] here.

Given a set of historical trajectories along with pertinent weather observations, the system works based upon an assumption that the weather observations are realizations of hidden aircraft positions, i.e., trajectory segments and the transitions between the underlying hidden segments following a Hidden Markov model [43]. This assumption considers a finite set of states, each of which is associated with a probability distribution over all possible trajectory segments. Transitions among the states are managed by a set of probabilities. The states are not visible, but the pertinent observations are. Given a sequence of observations, the system trains an HMM, a statistical Markov model, and derives a sequence of hidden states, aircraft positions that correspond to the sequence of weather observations. The system computes the most likely sequence of aircraft positions in three steps:

- In the training data processing step, the system transforms raw trajectories into aligned trajectories and combines weather parameters for each grid point along aligned trajectories. To achieve this, the system uses a 3D grid network with a spatial resolution of $6 \mathrm{~km} \times 6 \mathrm{~km}$ as a reference system.

- In the test data processing step, the system resamples the weather parameters to generate buckets with distinct ranges and feeds them into the time series clustering algorithm [10] to produce input observations.

- In the final step, the HMM parameters generated in the first two steps and the flight time computed by our Estimated Time of Arrival (ETA) Prediction System [5] are used as input to the Viterbi algorithm. The output is the optimal state sequence, joint $4 \mathrm{D}$ cubes defining aircraft trajectories.

During the conflict resolution stage, our current framework makes use of the first two steps, outlined above. However, unlike the third step of the process, our current framework avoids the cubes where the conflict is detected. This translates to a perturbation of at least one of the trajectories. Hence, the framework prescribes an optimized solution by perturbing at least one of the $4 \mathrm{D}$ trajectories, involved in the conflict. The process executes as follows: In addition to the regular HMM parameters of transition, emission, and initial probabilities, the framework uses conflict-free probabilities where each state is assigned a probability value indicating how conflictfree it is. The parameters are fed into a variant of the Viterbi Algorithm, in which the framework 
computes the optimal state sequence by considering the maximum HMM probabilities. Due to fact that the trajectory segments that are part of the first aircraft's trajectory are assigned low conflict-free probabilities, the framework avoids selecting them during the Viterbi process, yielding a conflict-free trajectory for the second aircraft.

Now, we present a variant of the Viterbi algorithm. Note that our previous Trajectory Prediction System [9] characterized an HMM by the following elements:

- $N$, the number of states in the model. States $S=\left\{S_{1}, S_{2}, \ldots, S_{N}\right\}$ are represented by reference points' coordinates (latitude, longitude, altitude) that form aligned trajectories. We denote state at time $t$ as $q_{t}$.

- $M$, the number of distinct observation symbols per state. Observations $V=\left\{v_{1}, v_{2}, \ldots, v_{M}\right\}$ are represented by weather parameters (temperature, wind speed, wind direction, humidity) recorded at grid points.

- The state transition probability distribution $A=\left\{a_{i j}\right\}$ is the probability of an aircraft discretely transitioning from one state $i$ to another $j$ along its aligned trajectory, where

$$
a_{i j}=P\left[q_{t+1}=S_{j} \mid q_{t}=S_{i}\right], \quad 1 \leq i, j \leq N .
$$

- The observation symbol probability distribution in state $j, B=b_{j}(k)$ is the probability of discrete weather parameters having been observed at that specific state, where

$$
b_{j}(k)=P\left[v_{k} \text { at } t \mid q_{t}=S_{j}\right], \quad \begin{gathered}
1 \leq j \leq N \\
1 \leq k \leq M
\end{gathered} .
$$

- The initial state distribution $\pi=\left\{\pi_{i}\right\}$ is the probability of an aligned trajectory beginning at a state $i$, where

$$
\pi_{i}=P\left[q_{1}=S_{i}\right], \quad 1 \leq i \leq N .
$$

These parameters form an HMM, compactly denoted by $\lambda=\{A, B$, and $\pi\}$. Now, we propose an additional parameter;

- The conflict-free probability distribution in state $j, C=c_{j}(k)$ is the probability of a conflict not occurring at that specific state, where

$$
c_{j}(k)=P\left[v_{k} \text { at } t \mid q_{t}=S_{j}\right], \quad \begin{gathered}
1 \leq j \leq N \\
1 \leq k \leq M
\end{gathered} .
$$

Hence, the lower the conflict-free probability for a particular state, the lower likelihood of that state to be included in the most probable path. With this new parameter, $C$ an HMM can be expanded and denoted by $\lambda=\{A, B, \pi$ and $C\}$.

The next step in the process is to choose a corresponding state sequence $Q=q_{1}, q_{2}, \ldots, q_{T}$ that best explains the observation sequence $O=O_{1}, O_{2}, \ldots, O_{T}$ given the model $\lambda$. A variant of the Viterbi algorithm [63] that is based on dynamic programming addresses this problem. The key component in the algorithm is the optimal probability, $\delta_{t}(j)$, and is computed as follows:

$$
\delta_{t}(j)=\max _{q_{1}, \ldots, q_{t-1}} \pi_{q_{1}} b_{q_{1}}\left(o_{1}\right) c_{q_{1}}\left(o_{1}\right) \prod_{j=2}^{t}\left(a_{q_{j-1}, q_{j}} b_{q_{j}}\left(o_{j}\right) c_{q_{j}}\left(o_{j}\right)\right) .
$$

Due to their low conflict-free probabilities, a variant of the Viterbi algorithm avoids trajectory segments where the conflict is detected, and generates a new optimized path. 


\subsection{CDR for Multiple Aircraft}

Our pairwise CDR solution can be scaled to address CDR for multiple aircraft that can be used towards better planning of airspace sector densities. Similar to the current flight planning procedures, we propose predicted trajectories to be processed on a first come first served basis. For the sake of simplicity, consider an empty airspace. The airspace will be fully available to the first predicted trajectory. Hence, the first trajectory's optimal set of data cubes will be reserved in the airspace. This process will introduce a set of constraints in the form of $P Z s$ by the first trajectory to the second and following trajectories during its flight time. Any conflict between the first and second trajectory will be detected and resolved. Once resolved, a new set of constraints will be introduced by the second trajectory. The next trajectory will need to satisfy the constraints introduced by the previous trajectories and so on. This also means that the next trajectory will need to avoid the $P Z s$ in the constraints list while generating its optimal set of data cubes during the conflict resolution phase. This process is repeated until one or more flights land, which will result in the removal of all pertinent constraints from the list, which will free up the particular sections of airspace.

Formally, given a new predicted trajectory $T_{j}=\left[p_{j 1}, p_{j 2}, \ldots, p_{j l}\right]$ and a time series of existing constraints list $C L_{P Z}=\left[P Z_{1}, P Z_{2}, \ldots, P Z_{i}, \ldots, P Z_{m}\right]$, where $P Z_{i}=\left[p z_{i 1}, p z_{i 2}, \ldots, p z_{i k}\right]$ along their existing trajectories $T=\left[T_{1}, T_{2}, \ldots, T_{i}, \ldots, T_{n}\right]$, where $T_{i}=\left[p_{i 1}, p_{i 2}, \ldots, p_{i k}\right]$, we want to detect and resolve conflicts among these trajectories $T_{j}$ and $T$ (i.e. one $v s$. all). Note that $P Z_{i}$ is formed by a set of data cubes, each defined by $4 \mathrm{D}$ spatio-temporal parameters around its centroid $p_{i j}$. With this approach, the implementation and management of the constraints list is of central importance. In our implementation, we map time instances to data cubes forming $P Z s$, which are stored in an Octree data structure, where there is one Octree for each time instance. Hence, when a new trajectory comes in, the pertinent Octree is located based on the trajectory's time instance. We declare a conflict if the trajectory segment is found in the Octree. We process all pairs of possibly conflicting data cubes using spatial indexing techniques to prune the search (e.g., References $[46,48]$ ).

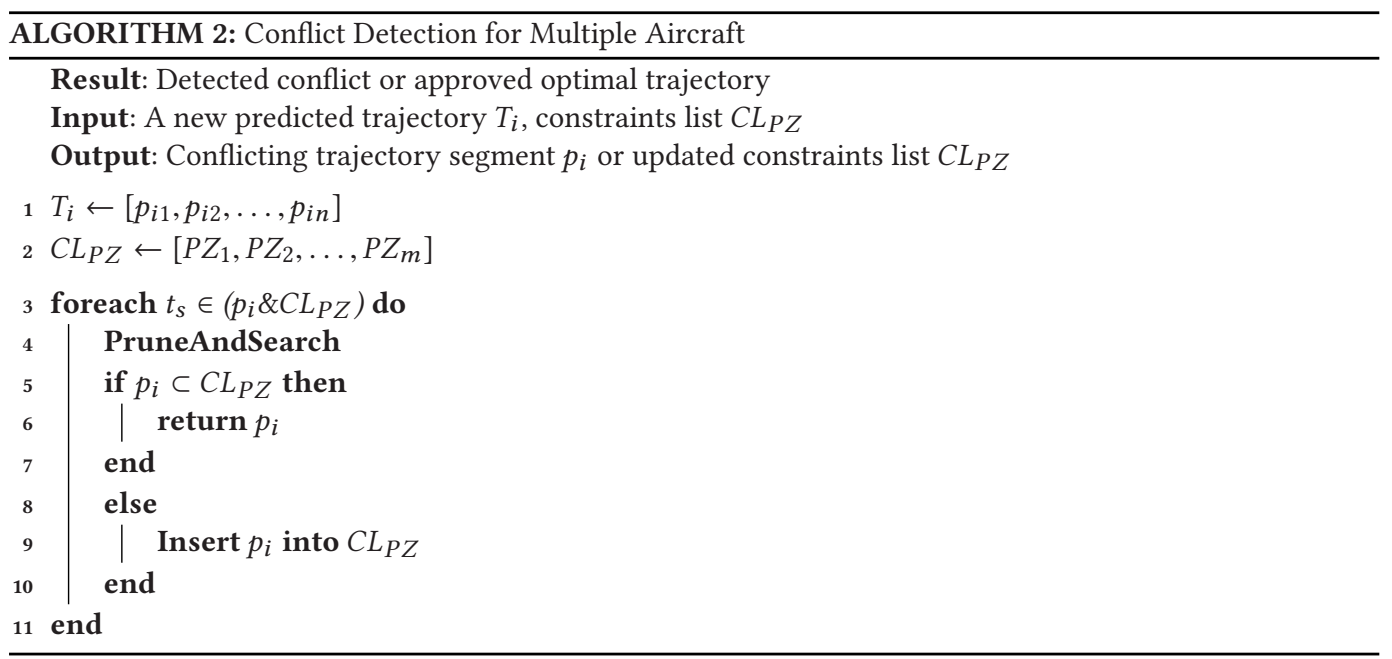

Our scalable conflict detection algorithm for multiple aircraft is presented in Algorithm 2. To detect a conflict, Algorithm 2 performs time instance lookup in constant time $O(1)$ for each trajectory segment. Next, Algorithm 2 performs a lookup operation for each trajectory segment in 
each Octree that has been created for each time instance, which have a running time of $O(\log n)$, where $n$ is the number of trajectory segments in the pertinent Octree. Given a new trajectory with $k$ segments, the worst-case running time of Algorithm 2 is $O(k * \log n)$, as the conflict is declared as soon as a new trajectory segment is found in any Octree for $k$ time instances. Here and in the rest of this work, we assume that the Octree is balanced.

To keep the constraints list up-to-date, we periodically check and delete the pertinent Octree if any of the time instances has expired. Once a conflict has been detected, a variant of the Viterbi algorithm is performed where all the data cubes included in the constraints list for the pertinent time instance are avoided to find a conflict-free, optimized path for the new trajectory. To achieve this, we assign a minimum conflict-free probability value, such as $1 \times 10^{-100}$ to those data cubes included in the constraints list.

Figure 4 is an illustration of a simplified case where two consecutive trajectory segments of aircraft \#1 and \#2 are inserted into two separate Octree data structures at time instances $t$ and $t+1$. For the sake of simplicity, the size of aircraft \#1's $P Z$ is considered as a single data cube. Assuming an empty airspace, the very first $P Z$ set for aircraft \#1 is allocated in the airspace without being considered for a potential conflict. This translates to the fact that an Octree for time instance $t$ and another Octree for time instance $t+1$ are created as they don't exist yet. Next, given the predicted trajectory for aircraft \#1, each consecutive $P Z$ in solid red is inserted into each Octree at time instance $t$ and $t+1$ as shown in Figure 4(a) and (b), respectively. When the predicted trajectory segments of aircraft \#2 in solid blue in Figure 4(c), and (d) are received, the Octree data structure at each time instance $t$ and $t+1$ are searched to see if the pertinent Octree node is empty. To achieve this, each Octree is looked up based on its index. Next, the pertinent Octree is searched as each time instance is mapped to an Octree. If found empty, then each consecutive trajectory segment is inserted into each Octree at time instance $t$ and $t+1$, as shown in Figure 4(c) and (d), respectively. The insert operation for the first trajectory segment of aircraft \#2 in solid blue is performed by traversing the node $k$ at depth $n$ illustrated as white cube mesh, followed by the node 0 at depth $n+1$ illustrated as yellow cube mesh, followed by the node 7 at depth $n+2$ illustrated as green cube mesh, and finally reaching to the leaf node 7 at depth $n+3$ and populating it with solid blue as shown in Figure 4(c). Upon search, if the leaf node is not found empty, which translates to a conflict, our conflict resolution Algorithm 3 is executed to perturb the predicted trajectory for aircraft \#2 to find an alternative optimal solution that results in conflict-free trajectories.

Formally, given the number of states $N$, number of distinct observations per state $M$, state transition probability distribution $A$, observation probability distribution $B$, and initial state probability distribution $\pi$, along with a conflict-free probability distribution for all data cubes included in the constraints list at time instances $t=\left[t_{1}, t_{2}, \ldots, t_{n}\right]$, we want to form an HMM $\lambda$, and train it to find the optimized path that is conflict-free. Our conflict resolution algorithm for multiple aircraft is presented in Algorithm 3.

To resolve a conflict, Algorithm 3 assigns a conflict-free probability of $1 \times 10^{-100}$ to each state in the state space when a trajectory segment in the new trajectory is found in any Octree that exists for $k$ time instances (i.e., the number of segments in the new trajectory), which has a running time of $O(k * \log n)$. Algorithm 3 assigns a conflict-free probability of 1 to the remaining states in the state space, which has a running time of $O(N-k)$, where $N$ is the number of states in the state space. Hence, the running time of Algorithm 3 between line 3 and line 11 is $O(k * \log n+N-k)$. Algorithm 3 performs a variant of the Viterbi algorithm between line 12 and line 13, which has a running time of $O\left(k *\left|N^{2}\right|\right)$. Last, Algorithm 3 inserts conflict-free trajectory segments into the constraints list between line 14 and line 16, which has a running time of $O(k * \log n)$. Hence, the total running time of Algorithm 3 is $O(k(2 \log n+|N|-1)+N)$. 


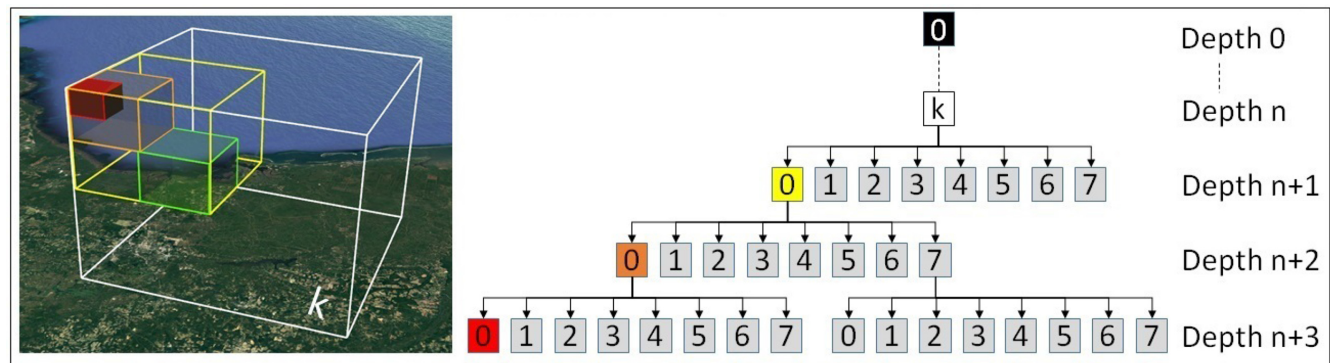

a) A single $P Z$ in solid red in OCTREE at time instance $t$.
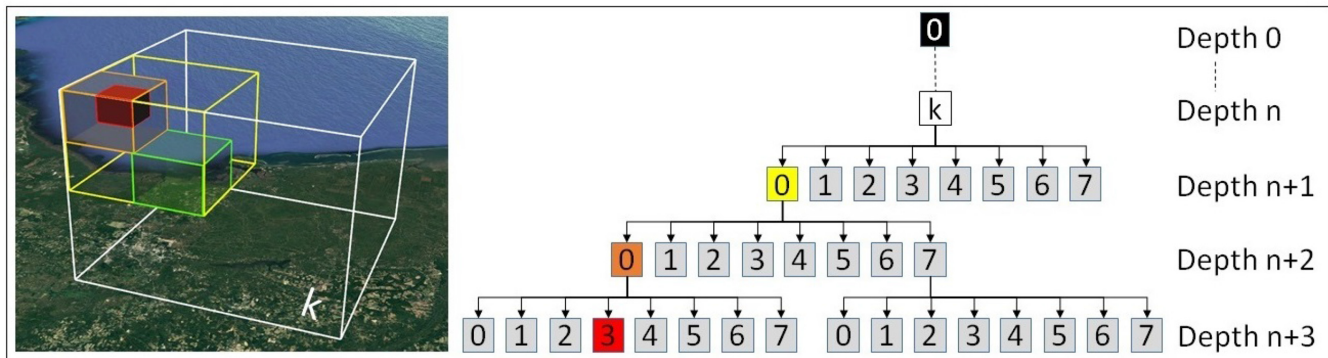

b) A single $P Z$ in solid red in OCTREE at time instance $t+1$.

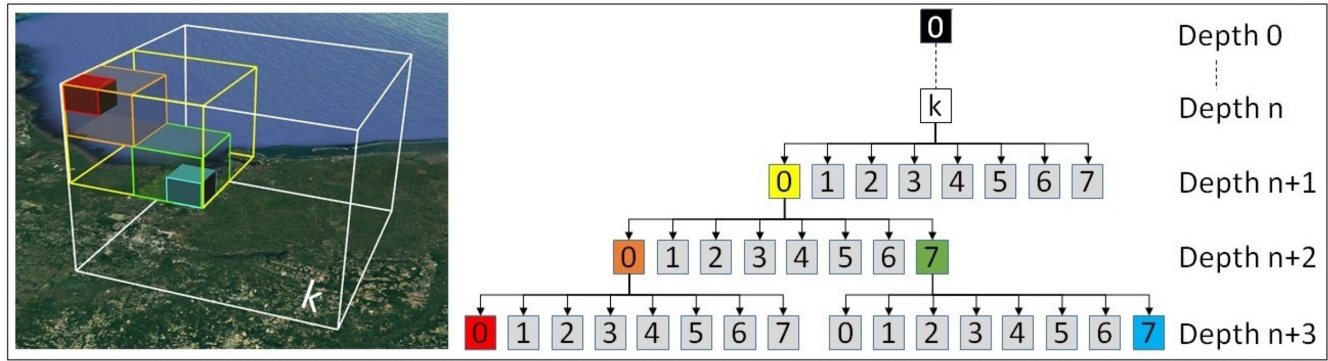

c) $P Z$ in solid red for aircraft \#1 and $P Z$ in solid blue for aircraft \#2 in OCTREE at time instance $t$.

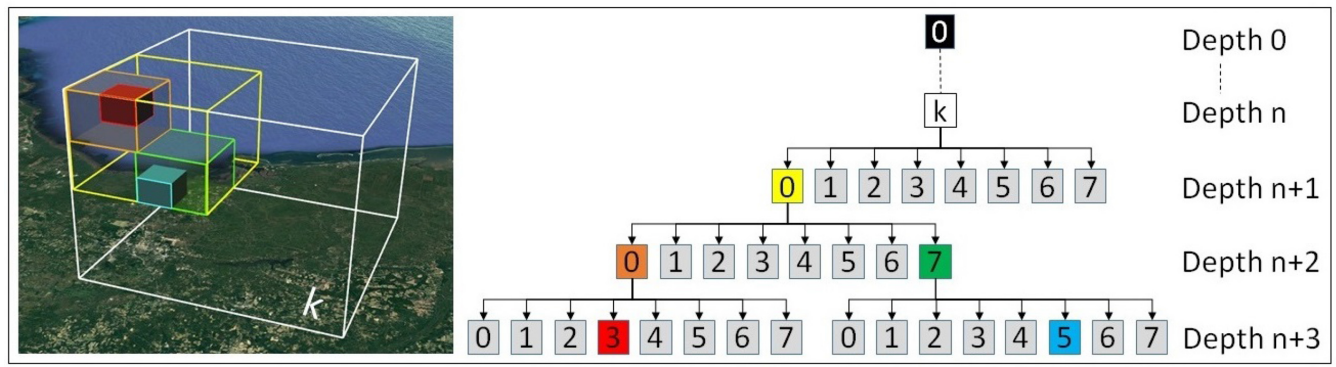

d) $P Z$ in solid red for aircraft \#1 and $P Z$ in solid blue for aircraft \#2 in OCTREE at time instance $t+1$.

Fig. 4. Octree data structure for efficient aircraft conflict detection and resolution. 


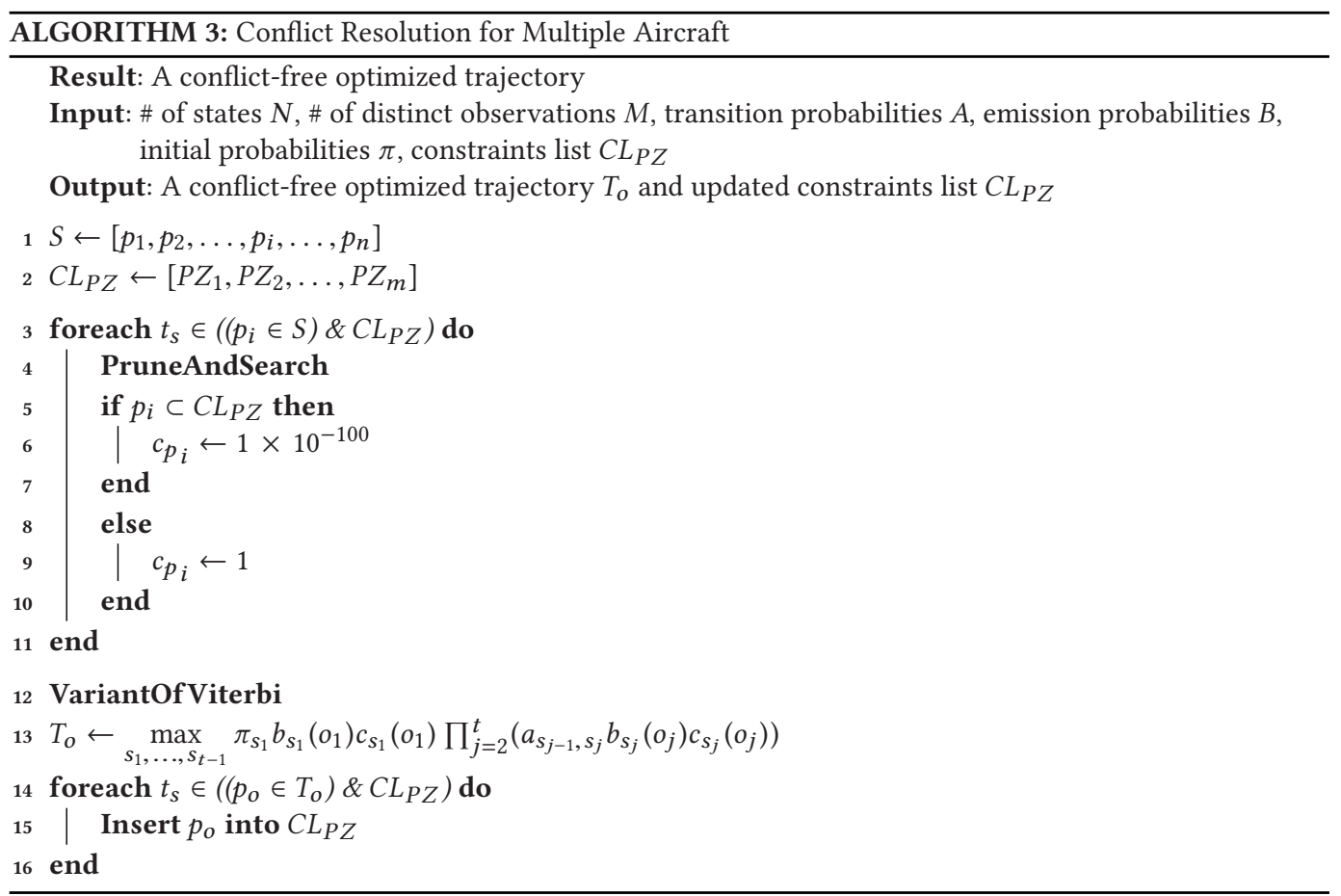

\section{EVALUATION}

To evaluate our framework, we generated a number of test cases using real trajectory and weather data from Europe and USA. Table 1 shows the European and USA airports forming the routes we used in our evaluation. Although the ideal evaluation would use real trajectories in actual conflicts, this is infeasible due to the nature of ATC operations, where the controller would interfere and separate the aircraft as soon as they are likely to infringe upon one another, so there would be no conflicts to find. Hence, we used a total of 24 test cases formed by European and USA flights that were in close proximity to cause potential conflicts when a minimal perturbation was applied. 16 of these 24 cases were used to test pairwise CDR, while 8 cases were used to test CDR for multiple aircraft.

\subsection{Setup}

Due to the fact that there exists no system that continuously records and stores exact positions of an aircraft's original trajectory [8], only a discrete set of sample data are recorded and stored, which presumably represent a close approximation of the original trajectory. We call this a raw trajectory. The raw trajectory data from Europe was provided by Spanish ANSP, ENAIRE using a radar surveillance feed with a $5 \mathrm{~s}$ update rate. The raw data was wrangled as part of the Data-driven AiRcraft Trajectory prediction research (DART) project under the SESAR Joint Undertaking Work Programme [56]. The European trajectory data contains all commercial domestic flights for Spain, a total of 119,563 raw trajectories and 80,784,192 raw trajectory points for the period of January through November 2016. The fields of the raw trajectory data are as follows: flight no, departure airport, arrival airport, date, time, aircraft speed in $X, Y, Z$ directions, and position information (latitude, longitude, altitude). Note that, as a preprocessing step, we downsampled raw trajectory data from the original resolution of $5 \mathrm{~s}$ to $60 \mathrm{~s}$ and aligned them to our 3D reference grid [9]. The raw trajectory data from the USA was extracted from an Aircraft Situation Display to Industry (ASDI) data 
Table 1. A Set of European and U.S.A. Airports

\begin{tabular}{l|l}
\hline Airport Code & \multicolumn{1}{c}{ Airport Name } \\
\hline LEAL & Alicante-Elche Airport \\
\hline LEBL & Barcelona-El Prat Airport \\
\hline LECO & A Coruña Airport \\
\hline LEIB & Ibiza Airport \\
\hline LEMD & Adolfo Suárez Madrid-Barajas Airport \\
\hline LEMG & Málaga Airport \\
\hline LEMH & Menorca Airport \\
\hline LEPA & Palma de Mallorca Airport \\
\hline LEVC & Valencia Airport \\
\hline LEVX & Vigo-Peinador Airport \\
\hline LEZL & Seville Airport \\
\hline \hline KATL & Hartsfield-Jackson Atlanta International Airport \\
\hline KBOS & Boston Logan International Airport \\
\hline KDFW & Dallas/Fort Worth International Airport \\
\hline KJAX & Jacksonville International Airport \\
\hline KLGA & NewYork LaGuardia Airport \\
\hline KMIA & Miami International Airport \\
\hline KORD & Chicago O'Hare International Airport \\
\hline KPIT & Pittsburgh International Airport \\
\hline
\end{tabular}

feed, which is recorded once every 60s, provided in near realtime by the FAA [23] and stored in an aviation data warehouse $[4,7]$. The USA trajectory data contains flights between 8 major airports, a total of 4,628 raw trajectories and 450,919 raw trajectory points for the period of May 2010 through December 2015. The fields of the raw trajectory data are as follows: source center, date, time, aircraft Id, speed, latitude, longitude, and altitude. Both European and USA weather data were extracted from the Global Forecast System (GFS), provided by NOAA [34]. The original data has $28 \mathrm{~km}$ spatial and $6 \mathrm{~h}$ temporal resolution and it contains over 40 weather parameters including atmospheric, cloud and ground attributes for each grid point as part of its 3D weather model. Hence, for this study's geographic volume and time period of interest, over 160TB of weather data was collected.

Due to fact that our current framework aims at addressing the CDR problem before departure, at least a pair of predicted trajectories are needed as input. Hence, we used our previous system [9] to generate a pair set of predicted trajectories. Next, we searched and found a number of trajectory points between the two flights' trajectories in the first and second pair, where the date, latitude, longitude, and altitude values matched, and time mismatched. By perturbing one of the flights' departure time we virtually created conflicts, where both aircraft traversed the same trajectory point at the same time. Table 2 shows the final size of training and test data in number of trajectories (\#trjs) and points (\#pts).

To evaluate our framework on multiple CDR test cases, we generated a set of three flights that is a combination of the existing routes listed in Table 2. The additional test cases for multiple CDR with their final size of test data in number of trajectories (\#trjs) and points (\#pts) are listed in Table 3. Table 3 does not include training data for each test case as they are already included in Table 2. Note that test case \#22 and \#23 use two separate virtual conflicts in each test case, where each conflict is addressed in sequence, i.e., first the virtual conflict between route \#1 and route \#2 is detected and resolved, next the virtual conflict between route \#1 and \#3 is detected and resolved. Unlike test cases \#22 and \#23, test cases \#17 through \#21 and \#24 address multiple CDR all at once, 
Table 2. Sizes of Training and Test Datasets for Pairwise CDR

\begin{tabular}{|c|c|c|c|c|c|c|c|c|c|c|}
\hline \multirow{2}{*}{ TestCase\# } & \multirow{2}{*}{ loute\#1 } & \multicolumn{2}{|c|}{ TrainingSetSize } & \multicolumn{2}{|c|}{ TestSetSize } & \multirow{2}{*}{ Route\#2 } & \multicolumn{2}{|c|}{ TrainingSetSize } & \multicolumn{2}{|c|}{ TestSetSize } \\
\hline & & \#trjs & \#pts & \#trjs & \#pts & & \#trjs & \#pts & \#trjs & \#pts \\
\hline 1 & LEAL-LEBL & 1118 & 55116 & 200 & 9860 & LEMD-LEIB & 2572 & 125623 & 200 & 9769 \\
\hline 2 & LEAL-LEBL & 1118 & 55116 & 19 & 937 & LEMD-LEMH & 1056 & 68141 & 19 & 1226 \\
\hline 3 & EAL-LEBL & 118 & 55116 & 152 & 7493 & LEPA-LEMD & 5116 & 306128 & 152 & 9095 \\
\hline 4 & BL-LEMG & 704 & 7451 & 43 & 3216 & LEMD-LEMH & 1056 & 8141 & 43 & 2775 \\
\hline 5 & LEBL-LEMG & 1704 & 127451 & 180 & 13463 & LEPA-LEMD & 5116 & 306128 & 180 & 10771 \\
\hline 6 & LEBL-LEZL & 2404 & 183343 & 41 & 3127 & LEMI & 1434 & 70128 & 41 & 2005 \\
\hline 7 & EBL-LEZL & 2404 & 183343 & 46 & 3508 & LEMD-LEMH & 1056 & 68141 & 46 & 2968 \\
\hline 8 & LEBL-LEZL & 2404 & 183343 & 164 & 12508 & LEMG-LEMD & 1403 & 75408 & 164 & 8815 \\
\hline 9 & LEBL-LEZL & 2404 & 183343 & 210 & 16016 & LEPA-LEMD & 5116 & 306128 & 210 & 12566 \\
\hline 10 & LEIB-LEBL & 1360 & 53443 & 259 & 10178 & LEPA-LEMD & 5116 & 306128 & 259 & 15498 \\
\hline 11 & LEIB-LEBL & 1360 & 53443 & 158 & 6209 & LEPA-LEVC & 1426 & 50467 & 158 & 5592 \\
\hline 12 & LEMG-LEBL & 1563 & 114767 & 38 & 2790 & LEMD-LEAM & 1434 & 70128 & 38 & 1858 \\
\hline 13 & LEMG-LEBL & 1563 & 114767 & 46 & 3378 & LEMD-LEIB & 2572 & 125623 & 46 & 2247 \\
\hline 14 & LEZL-LEBL & 2380 & 186299 & 40 & 3131 & LEMD-LEIB & 2572 & 125623 & 40 & 1954 \\
\hline 15 & KDFW-KJAX & 1355 & 153970 & 19 & 2159 & KATL-KMIA & 1296 & 108005 & 19 & 1583 \\
\hline 16 & KLGA-KORD & 1155 & 131454 & 62 & 7056 & KPIT-KBOS & 822 & 57490 & 62 & 4339 \\
\hline
\end{tabular}

Table 3. Sizes of Training and Test Datasets for Multiple Aircraft CDR

\begin{tabular}{|c|c|c|c|c|c|c|c|c|c|}
\hline \multirow{2}{*}{ TestCase\# } & \multirow{2}{*}{ Route\#1 } & \multicolumn{2}{|c|}{ TestSetSize } & \multirow{2}{*}{ Route\#2 } & \multicolumn{2}{|c|}{ TestSetSize } & \multirow{2}{*}{ Route\#3 } & \multicolumn{2}{|c|}{ TestSetSize } \\
\hline & & \#trjs & \#pts & & \#trjs & $\# p t s$ & & \#trjs & \#pts \\
\hline 17 & LEAL-LEBL & 5 & 253 & LEMD-LEMH & 5 & 322 & LEMD-LEPA & 5 & 304 \\
\hline 18 & LEBL-LEMG & 3 & 226 & LEMD-LEMH & 3 & 197 & LEBL-LEZL & 3 & 233 \\
\hline 19 & LEBL-LEMG & 30 & 2244 & LEMD-LEMH & 30 & 1935 & LEMD-LEPA & 30 & 1795 \\
\hline 20 & LEBL-LEMG & 22 & 1672 & LEPA-LEMD & 22 & 1321 & LEBL-LEZL & 22 & 1694 \\
\hline 21 & LEBL-LEZL & 27 & 2059 & LEMD-LEMH & 27 & 1742 & LEMD-LEPA & 27 & 1616 \\
\hline 22 & LEBL-LEZL & 164 & 12508 & LEMG-LEMD & 164 & 8815 & LEPA-LEMD & 210 & 12566 \\
\hline 23 & LEMG-LEBL & 38 & 2790 & LEMD-LEAM & 38 & 1858 & LEMD-LEIB & 46 & 2247 \\
\hline 24 & LEMG-LEBL & 6 & 441 & LEMD-LEIB & 6 & 384 & LEMD-LEPA & 6 & 363 \\
\hline
\end{tabular}

i.e., all three routes share a common trajectory point. Hence, to form a set of three flights for test case \#17 through \#21 and \#24, we searched and found a number of trajectory points between these three flights' trajectories, where the date, latitude, longitude, and altitude values matched, and time mismatched. Assuming that the predicted trajectories were received in the order of route $\# 1$, \#2, and \#3, respectively, first we perturbed the second flights' departure time to create a virtual conflict between the first and second flight, which is detected and resolved using our multiple CDR approach. As an outcome, route \# 2 was perturbed at the conflict location, yielding a common trajectory point between route \#1 and route \#3. Next, we perturbed the third flights' departure time to create a virtual conflict between the first and third flight, which is also detected and resolved using our multiple CDR approach. In all multiple CDR test cases listed in Table 3, we used an Octree data structure and executed Algorithm 2 and Algorithm 3 as highlighted in Section 4.3.

Note that overall, the trajectory data alone contains over 4 million trajectory points. In Tables 2 and 3, the test data represents the conflicting trajectories. Aside from these 1,677 trajectory pairs, 


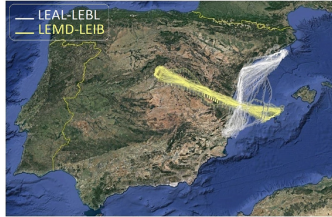

a) Test case \#1: LEAL-LEBL and LEMD LEIB.

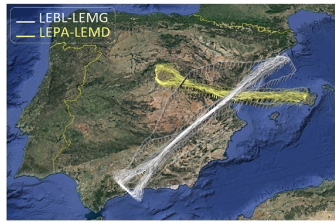

e) Test case \#5: LEBL-LEMG and LEPA-LEMD.

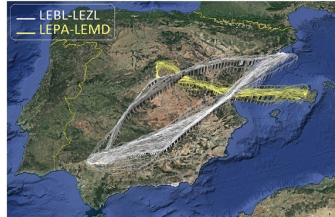

i) Test case \#9: LEBL-LEZL and LEPA-LEMD.

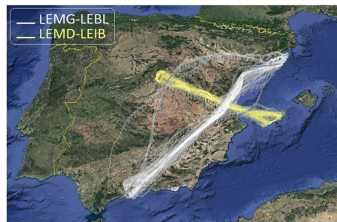

m) Test case \#13: LEMG-LEBL and LEMD-LEIB.

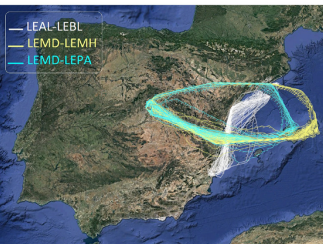

r) Test case \#17: LEAL-LEBL and

EMD-LEMH and LEMD-LEPA.

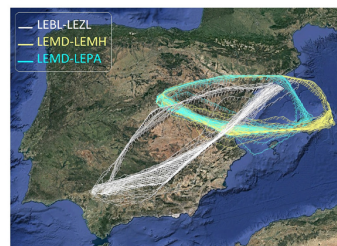

v) Test case \#21: LEBL-LEZL and LEMD-LEMH and LEMD-LEPA.
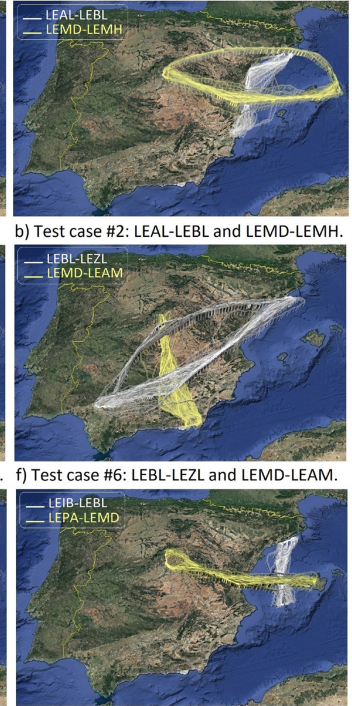

i) Test case \#10: LEIB-LEBL and LEPA-LEMD.
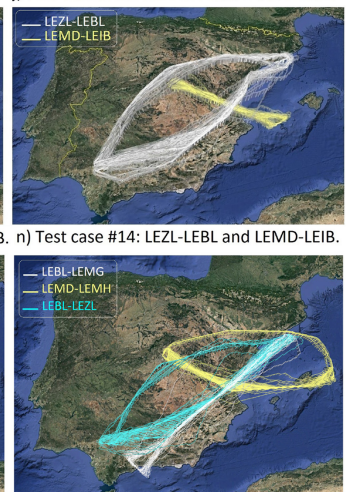

s) Test case \#18: LEBL-LEMG and LEMD-LEMH and LEBL-LEZL.

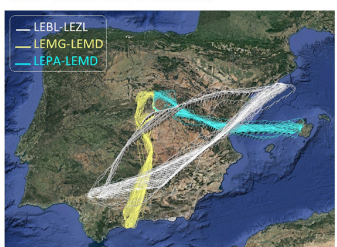
w) Test case \#22: LEBL-LEZL

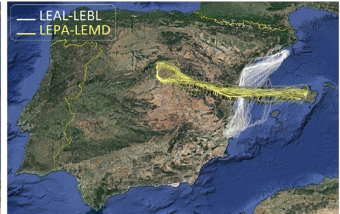

c) Test case \#3: LEAL-LEBL and LEPA-LEMD.

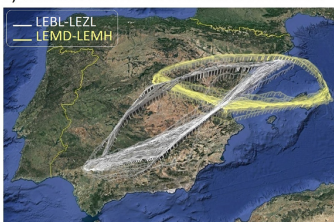

g) Test case \#7: LEBL-LEZL and LEMD-LEMH.

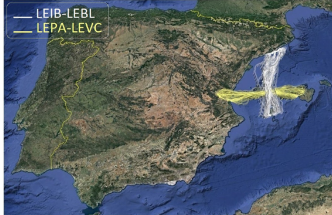

k) Test case \#11: LEIB-LEBL and LEPA-LEVC

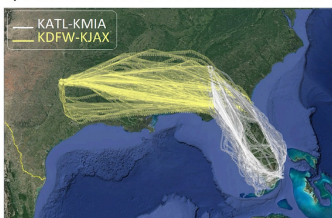

Test case H12: LEMG -LEBL and LEMD-LEAM.
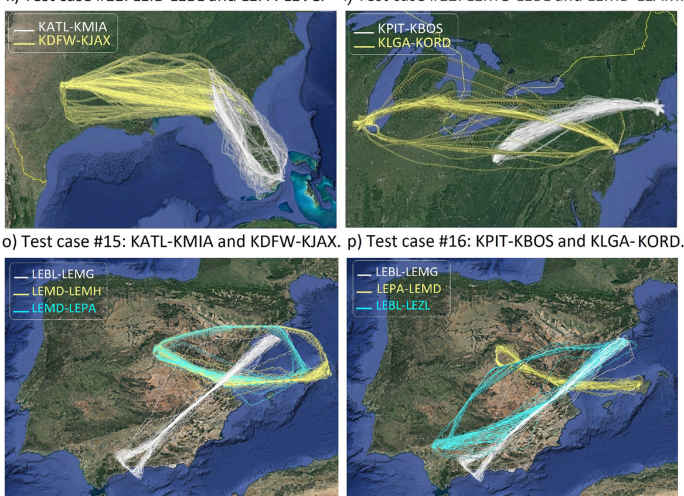

t) Test case \#19: LEBL-LEMG and LEMD-LEMH and LEMD-LEPA.

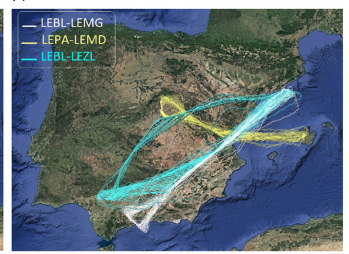

u) Test case \#20: LEBL-LEMG and LEPA-LEMD and LEBL-LEZL.
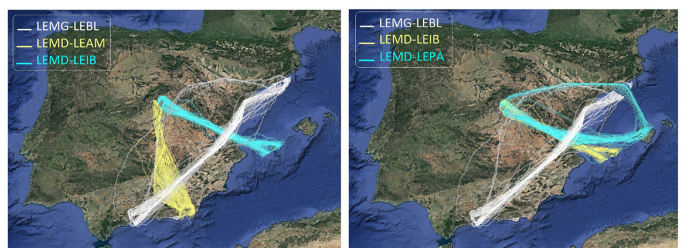

y) Test case \#24: LEMG-LEBL and LEMD-LEIB and LEMD-LEPA.

Fig. 5. Visual representation of training and test data for conflicting flights.

we also bootstrapped by drawing 100 additional trajectory pairs with replacement from the trajectory set to test for the false positive cases. Hence, we evaluated our framework's effectiveness with a total of 3,554 $(3,354+200)$ test trajectories on 16 test cases for pairwise CDR and an additional set of 1,234 test trajectories formed by three flights on 8 test cases for multiple CDR.

Figures 5(a) through (p) illustrate the pairwise test cases with their cumulative training and test data in white and yellow, respectively, whereas Figure 5(r) through (y) illustrate the test cases of three flights with their cumulative training and test data in white, yellow, and blue, respectively. 

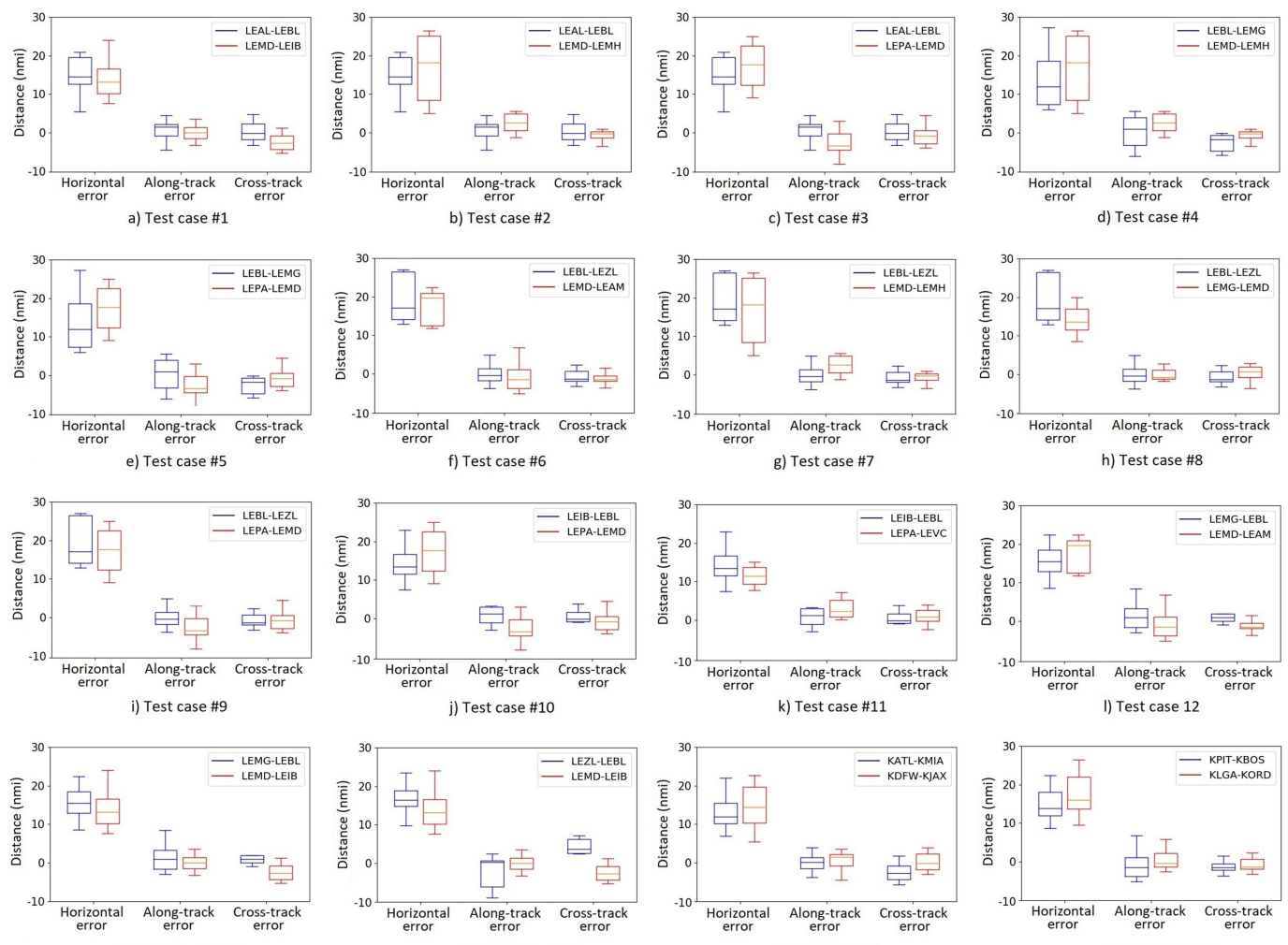

m) Test case \#13
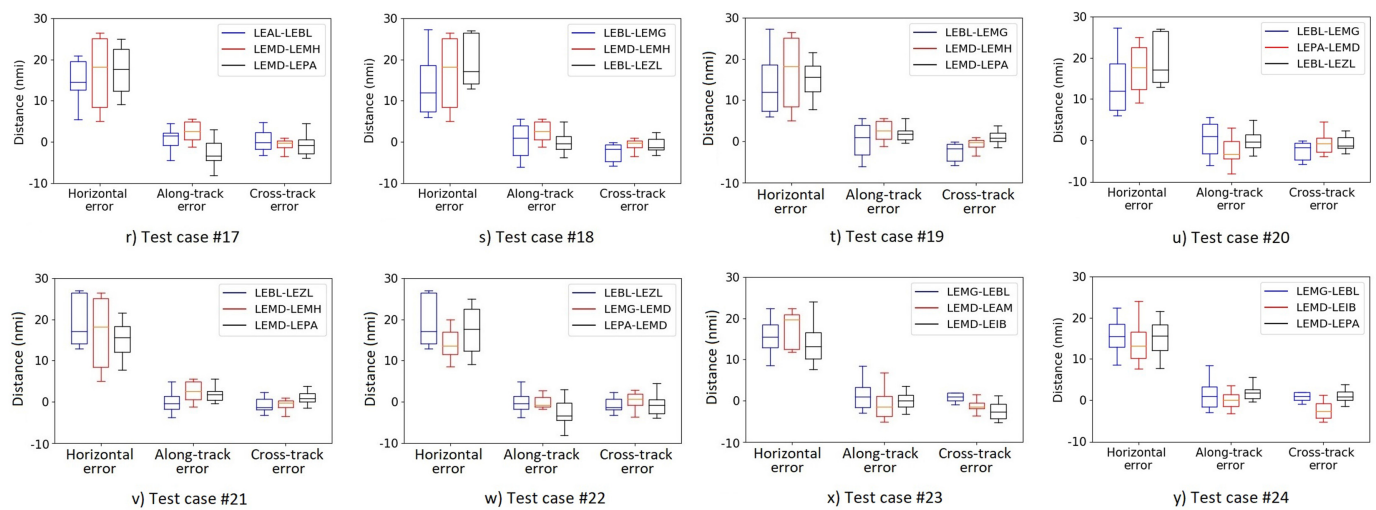

Fig. 6. Trajectory prediction errors in the form of box plots.

\subsection{Results}

We evaluated our framework by comparing the output of each step through the framework's pipeline with the ground truth on all 24 test cases. These steps include conflict detection and conflict resolution. However, due to fact that the accuracy of conflict detection and resolution significantly depend on the accuracy of predicted trajectories, we also computed trajectory prediction errors. For that purpose, we used trajectory prediction accuracy metrics as outlined in Reference [37] and computed horizontal and vertical errors $e_{\text {horiz }}, e_{\text {vert }}$ based on predicted trajectories generated by our previous system [9] versus raw trajectories of pertinent flights. The box plots in Figure 6 capture the aggregated errors for horizontal, along-track, and cross-track errors 
Table 4. Horizontal and Vertical Error Ranges for Each Bin Size

\begin{tabular}{c|c|c}
\hline BinSizeId & Condition & Size \\
\hline 1 & $0 \mathrm{nmi} \leq e_{\text {horiz }} \leq 5 \mathrm{nmi} \wedge 0 \mathrm{ft} \leq e_{\text {vert }} \leq 2000 \mathrm{ft}$ & $5 \mathrm{nmi}, 2000 \mathrm{ft}$ \\
\hline 2 & $5 \mathrm{nmi}<e_{\text {horiz }} \leq 10 \mathrm{nmi} \vee 2000 \mathrm{ft}<e_{\text {vert }} \leq 4000 \mathrm{ft}$ & $10 \mathrm{nmi}, 4000 \mathrm{ft}$ \\
\hline 3 & $10 \mathrm{nmi}<e_{\text {horiz }} \leq 15 \mathrm{nmi} \vee 4000 \mathrm{ft}<e_{\text {vert }} \leq 6000 \mathrm{ft}$ & $15 \mathrm{nmi}, 6000 \mathrm{ft}$ \\
\hline 4 & $15 \mathrm{nmi}<e_{\text {horiz }} \leq 20 \mathrm{nmi} \vee 6000 \mathrm{ft}<e_{\text {vert }} \leq 8000 \mathrm{ft}$ & $20 \mathrm{nmi}, 8000 \mathrm{ft}$ \\
\hline
\end{tabular}

for each flight in all 24 test cases. The horizontal error is unsigned whereas the along-track, crosstrack, and vertical errors are signed errors. Note that the mean value for the cross-track error and vertical error along the entire test trajectories are $7.693 \mathrm{nmi}$ and $1589.459 \mathrm{ft}$, respectively, when the sign is omitted.

To evaluate our conflict detection capability, we used the same accuracy metrics as in Reference [37] and computed horizontal and vertical errors by comparing the output of conflict detection and conflict resolution of our framework with the ground truth on all 24 test cases. To compute the errors, we fed a pair set of predicted trajectories for test case \#1 through \#16 and a set of three predicted trajectories for test case \#17 through \#24 into our conflict detection algorithm and compared the locations of virtual conflicts versus locations of conflicts detected by our framework. Next, we created 4 bin sizes, where each bin size is an integer multiple of $5 \mathrm{nmi}$ of lateral and 2,000ft of vertical distances, conventionally accepted as minimum separation values for enroute airspace by ANSPs.

Table 4 presents the pertinent condition for each bin. Using horizontal and vertical error values, we counted the number of conflicts in each case and found the bin size to which they belong. The outcome is presented as a set of histograms in Figure 7. Our algorithm detected $\mathbf{8 7 . 1 \%}$ of the conflicts within the first bin size and $\mathbf{9 9 \%}$ of the conflicts within the first two bin sizes on all 24 test cases. Note that the conflict detection can only be as accurate as the predicted trajectories. Hence, these errors are attributed to the accuracy of our previous system [9], defined by the horizontal,

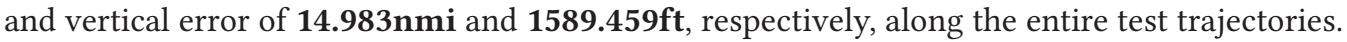

To resolve the conflicts, we ran our conflict resolution algorithm as highlighted in Section 4.2. Figure 8 provides a closer look at one of the detected and resolved conflicts by our framework for a pairwise CDR test case. In all figures, the yellow cubes and white cubes represent the first and second flight's, respectively, predicted trajectories. The red line parallel to the white cubes represents the first fight's actual trajectory and the red line parallel to the yellow cubes represents the second flight's actual trajectory. As both aircraft move one cube at a time in the flight direction, the $P Z$ illustrated in the cyan cube around the current position of the first flight also moves forward in the form of a sliding window. The first figure on the far left shows where each aircraft is at time $t_{s-1}$, represented respectively by the solid white cubes for the first and yellow cubes for the second flight. The second figure from the left captures the conflict detected at time interval $t_{s}$ by our framework illustrated with a solid red cube. The actual conflict occurs where the red lines intersect. Note that both the predicted conflict position and the actual conflict position are within the first flight's $P Z$. The third figure from the left is the 3D view of the second figure from the left. The actual and predicted conflict positions are only 2 cube sizes away from each other, considering the center of the cube as the predicted position of the aircraft. The figure in the far right illustrates the optimized solution to the conflict by our framework. The first flight's trajectory has been perturbed vertically and the conflict has been resolved by our framework. The altitude of the second flight has been elevated resulting in conflict being resolved. Note that due to assignment of low conflict-free probabilities to the 3D grid points inside of the $P Z$, the framework avoids selecting them during the conflict resolution stage, generating conflict free trajectories. 

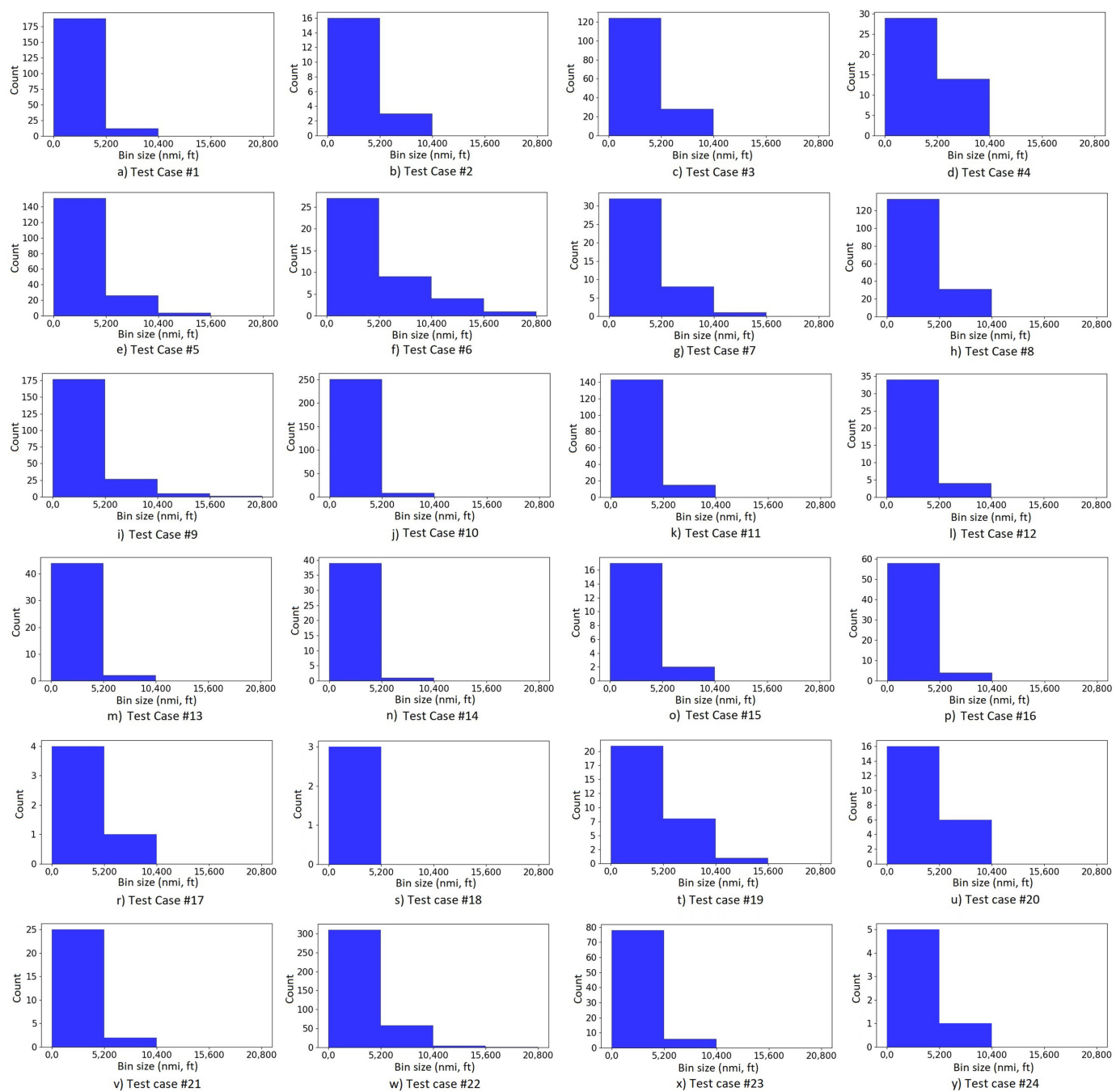

Fig. 7. Conflict detection histograms showing how many conflicts were captured by our framework in each bin size.

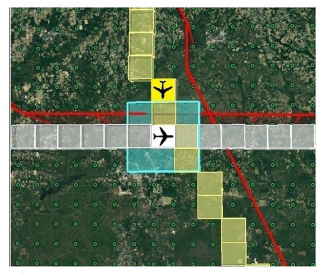

a) Aircraft at time $t_{s-1}$.

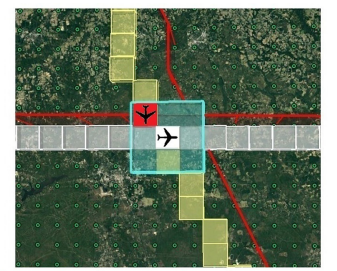

b) Predicted vs. actual conflict at time $t_{s}$. c) 3D view of the conflict.

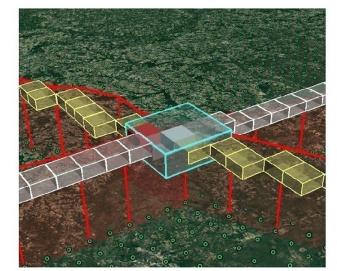

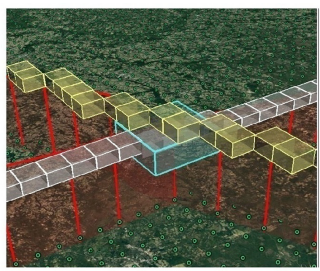

d) Optimized solution to the conflict.

Fig. 8. Illustration of a detected and resolved pairwise conflict by our framework. 
Table 5. Accuracy of Our Conflict Resolution Algorithm Based on Each Bin Size

\begin{tabular}{c|c|c}
\hline BinSizeId & Size & Accuracy \\
\hline 1 & $5 \mathrm{nmi}, 2000 \mathrm{ft}$ & $98.3 \%$ \\
\hline 2 & $10 \mathrm{nmi}, 4000 \mathrm{ft}$ & $97.5 \%$ \\
\hline 3 & $15 \mathrm{nmi}, 6000 \mathrm{ft}$ & $93.7 \%$ \\
\hline 4 & $20 \mathrm{nmi}, 8000 \mathrm{ft}$ & $100.0 \%$ \\
\hline
\end{tabular}

As the final evaluation step, we executed our conflict resolution algorithm on all conflicts formed by a total of $4,788(3,554+1,234)$ trajectories and computed accuracy values based on the number of successful resolutions, i.e. generating a feasible safe alternative trajectory by fulfilling the separation minima criteria. Due to the fact that our conflict resolution algorithm resolved the vast majority of conflicts on all test cases, we provide aggregated results overall, rather than provide results for each test case. Table 5 presents accuracy values for each bin size. Note that, to resolve the conflicts, we treated each bin size differently based on their varying sizes so that only relevant 3D grid points were avoided. The process perturbed the trajectory for the latter flight, generated an optimal alternative and yielded conflict-free trajectories.

\section{DISCUSSION}

During the trajectory prediction step, errors were computed by comparing the locations of virtual conflicts versus locations of conflicts detected by our framework. The trajectory prediction had

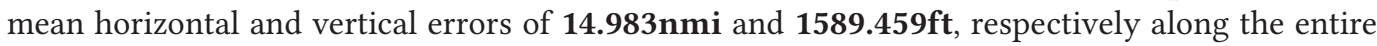
trajectory points over the full test set from two continents. Using the predicted trajectories, our conflict detection algorithm had $\mathbf{6 . 0 1 3} \mathbf{n m i}$ of mean horizontal error. Note that this value is considerably less than $14.983 \mathrm{nmi}$, the mean horizontal error by our previous Trajectory Prediction System [9] along the entire trajectory points including climb, cruise and descent phases of a flight. This is due to two major facts: (1) Trajectory prediction accuracy during the cruise phase is often considerably higher than the climb and descent phases of the flight. (2) All conflicts we used in our experiments took place during the cruise phase of the flights. With $6.013 \mathrm{nmi}$ of mean horizontal error on the conflict positions, our conflict detection algorithm found $\mathbf{9 9 \%}$ of conflicts within the first two bin sizes of separation minima $(10 \mathrm{nmi}, 4000 \mathrm{ft})$. We also verified that between none of the additional 100 trajectory pairs where the virtual conflict never occurred was falsely detected as a conflict by our framework. Our conflict resolution algorithm's mean accuracy was over $\mathbf{9 7 \%}$ on all test cases. Though, it is interesting to see that it was unable to reach $100 \%$ accuracy, given the fact that all it had to do was avoid the selected 3D grid points when generating the new conflict-free optimal trajectory. The reason for that was the sparse distribution of data cubes, i.e., lack of data over the 3D grid network. The algorithm was unable to connect the new trajectory segments from start to end due to disconnection. Hence, our framework craves for more data to reach higher accuracy values.

These results validate the effectiveness of our framework on the long-range CDR problem. However, this is not to say that strategic CDR will detect and resolve conflicts once for all and no more conflicts will occur during the flight. There will likely be some convective weather patterns shaping after departure. These sudden changes causing potential conflicts should be addressed tactically by short and or medium-range CDR systems while the aircraft is airborne.

Although we were not able to find, there may also likely be some exceptional cases where false positive conflicts may be found. These cases should also be addressed tactically by short and or 
medium-range CDR systems. Note that the larger the selected $P Z$ value, the higher probability of finding and resolving the conflicts between trajectories. However, that also means less denser sectors resulting in inefficient use of airspace. Hence, the tradeoff should be handled carefully by the ANSPs.

Overall, we propose our framework to be used by AOCs to file more realistic flight plans. Our framework can also be used by ANSPs to validate that the filed flight plans do not cause conflicts with previously approved flight plans or increase any sector traffic complexities. Air traffic density and complexity (a count of aircraft predicted to be in conflict with another) are two major factors defining the metric of air traffic controller workload [28]. These goals can be achieved in the planning phase before the aircraft depart, resulting in improvement in the four ATM key performance areas, safety, capacity, efficiency, and environmental impact, and thereby improving ATM automation and reducing the air traffic controller workload.

\section{CONCLUSION}

We have presented a novel Data-driven Framework addressing a long-range CDR problem. Using a set of predicted trajectories, the framework delivers two major capabilities; (i) conflict detection and (ii) conflict resolution before the flight depart. In the conflict detection stage, the framework declares a conflict when a $P Z$ of an aircraft on its predicted trajectory is infringed upon by another aircraft at the same time interval in the future. In the conflict resolution stage, upon a conflict, the framework executes our conflict resolution algorithm that is derived from the Viterbi algorithm and prescribes a solution that resolves the conflict by perturbing at least one of the $4 \mathrm{D}$ trajectories.

Our experiments on real trajectory and weather datasets from two continents verify that our framework achieves lateral and vertical accuracies that are within the boundaries of conventionally accepted minimum separation values, set by the ANSPs. This translates to the fact that, ANSPs can now detect and resolve poten tial conflicts long before the aircraft depart, resulting in safer and greener skies with higher efficiency and capacity, and thereby reducing the air traffic controller workload. Some future work could involve adding a spatial browsing capability [12, 19, 47] for the trajectories as well as incorporating our methods in a distributed spatial environment [60].

\section{REFERENCES}

[1] A. Amir, A. Efrat, P. Indyk, and H. Samet. 1999. Efficient regular data structures and algorithms for location and proximity problems. In Proceedings of the 40th IEEE Annual Symposium on Foundations of Computer Science. 160-170.

[2] A. Amir, A. Efrat, P. Indyk, and H. Samet. 2001. Efficient algorithms and regular data structures for dilation, location and proximity problems.Algorithmica 30, 2 (Jun. 2001), 164-187.

[3] C. H. Ang, H. Samet, and C. A. Shaffer. 1990. A new region expansion for quadtrees. IEEE Trans. Pattern Anal. Mach. Intell. 12, 7 (Jul. 1990), 682-686.

[4] S. Ayhan, P. Comitz, and V. Stemkovski. 2009. Aviation mashups. In Proceedings of the IEEE/AIAA 28th Digital Avionics Systems Conference. 6.D.5-1-6.D.5-9.

[5] S. Ayhan, P. Costas, and H. Samet. 2018. Predicting estimated time of arrival for commercial flights. In Proceedings of the 24nd ACM SIGKDD International Conference on Knowledge Discovery and Data Mining. 33-42.

[6] S. Ayhan, P. Costas, and H. Samet. 2018. Prescriptive analytics system for long-range aircraft conflict detection and resolution. In Proceedings of the 26th ACM SIGSPATIAL International Conference on Advances in Geographic Information Systems.

[7] S. Ayhan, J. Pesce, P. Comitz, G. Gerberick, and S. Bliesner. 2012. Predictive analytics with surveillance big data. In Proceedings of the 1st ACM SIGSPATIAL International Workshop on Analytics for Big Geospatial Data.

[8] S. Ayhan and H. Samet. 2015. DICLERGE: Divide-cluster-merge framework for clustering aircraft trajectories. In Proceedings of the 8th ACM SIGSPATIAL International Workshop on Computational Transportation Science.

[9] S. Ayhan and H. Samet. 2016. Aircraft trajectory prediction made easy with predictive analytics. In Proceedings of the 22nd ACM SIGKDD International Conference on Knowledge Discovery and Data Mining. 21-30. 
[10] S. Ayhan and H. Samet. 2016. Time series clustering of weather observations in predicting climb phase of aircraft trajectories. In Proceedings of the 9th ACM SIGSPATIAL International Workshop on Computational Transportation Science. 25-30.

[11] K. Bilimoria, B. Sridhar, and G. Chatterji. 1996. Effects of conflict resolution maneuvers and traffic density on free flight. In Proceedings of the AIAA GNC Conference and Exhibit. 1-11.

[12] F. Brabec and H. Samet. 2007. Client-based spatial browsing on the world wide web. IEEE Internet Comput. 11, 1 (Jan./Feb. 2007), 52-59.

[13] D. J. Brudnicki, K. S. Lindsay, and A. L. McFarland. 1997. Assessment of field trials, algorithmic performance, and benefits of the user request evaluation tool (URET) conflict probe. In Proceedings of the 16th DASC. AIAA/IEEE Digital Avionics Systems Conference. 35-44.

[14] B. Carpenter and J. Kuchar. 1997. Probability-based collision alerting logic for closely-spaced parallel approach. In Proceedings of the 35th Aerospace Sciences Meeting and Exhibit. 1-8.

[15] J. Cobano, D. Alejo, G. Heredia, and A. Ollero. 2013. 4D trajectory planning in ATM with an anytime stochastic approach. In Proceedings of the 3rd International Conference on ATACCS. 1-8.

[16] J. A. Cobano, D. Alejo, A. Ollero, and A. Viguria. 2012. Efficient conflict resolution method in air traffic management based on the speed assignment. In Proceedings of the 2nd International Conference on Application and Theory of Automation in Command and Control Systems. 54-61.

[17] G. Dowek and C. Munoz. 2007. Conflict detection and resolution for 1,2, .,N aircraft. In Proceedings of the 7th AIAA Aviation Technology, Integration and Operations Conference. 1-13.

[18] V. N. Duong and E. G. Hoffman. 1997. Conflict resolution advisory service in autonomous aircraft operations. In Proceedings of the 16th DASC. AIAA/IEEE Digital Avionics Systems Conference. 10-17.

[19] C. Esperança and H. Samet. 2002. Experience with SAND/Tcl: A scripting tool for spatial databases. F. Vis. Lang. Comput. 13, 2 (Apr. 2002), 229-255.

[20] FAA. 2009. The NextGen. Retrieved from https://www.faa.gov/nextgen/.

[21] FAA. 2012. Traffic Alert and Collision Avoidance System (TCAS II). Retrieved from https://www.faa.gov/other_visit/ aviation_industry/airline_operators/airline_safety/info/all_infos/media/2012/InFO12010.pdf.

[22] FAA. 2015. TAF. Retrieved from http://taf.faa.gov/Downloads/TAFSummaryFY2015-2040.pdf.

[23] FAA. 2016. Aircraft Situation Display to Industry. Retrieved from http://www.fly.faa.gov/ASDI/.

[24] G. B. M. Heuvelink and H. A. Blom. 1998. An alternative method to solve a variational inequality applied to an air traffic control example. In Analysis and Optimization of Systems. Springer-Verlag, London, UK, 617-628.

[25] R. Irvine. 1998. GEARS conflict resolution algorithm. In Proceedings of the AIAA GNC Conference and Exhibit. 786-796.

[26] E. Jacox and H. Samet. 2008. Metric space similarity joins. ACM Trans. Database Syst. 33, 2 (Jun. 2008), 7.

[27] J. Kuchar and L. Yang. 2000. A review of conflict detection and resolution modeling methods. IEEE Trans. Intell. Transport. Syst. 1, 4 (Dec. 2000), 179-189.

[28] I. V. Laudeman, S. G. Shelden, R. Branstrom, and J. L. Brasil. 1998. Dynamic Density: An Air Traffic Management Metric. Technical Report. NASA Ames Research Center, Mountain View, CA.

[29] T. Lauderdale. 2012. Probabilistic conflict detection for robust detection and resolution. In Proceedings of the 12th AIAA ATIO Conference and 14th AIAA/ISSMO Multidisciplinary Analysis and Optimization Conference. 1-12.

[30] W. Liu and I. Hwang. 2011. Probabilistic trajectory prediction and conflict detection for air traffic control. F. Guid. Contr. Dynam. 34 (Nov. 2011), 1779-1789.

[31] Y. Matsuno. 2013. Probabilistic Conflict Detection in the Presence of Uncertainty. Springer Japan, Tokyo, 17-33.

[32] D. McNally, R. Bach, and W. Chan. 1998. Field test evaluation of the CTAS conflict prediction and trial planning capability. In Proceedings of the AIAA GNC Conference and Exhibit. 1686-1697.

[33] P. Menon, G. Sweriduk, and B. Sridhar. 1999. Optimal strategies for free-flight air traffic conflict resolution. F. Guid. Contr. Dynam. 22 (Mar. 1999), 202-211.

[34] NOAA. 2016. NCEP Global Forecast System. Retrieved from https:/www.ncdc.noaa.gov/data-access/model-data/ model-datasets/global-forcast-system-gfs.

[35] S. Nutanong, E. H. Jacox, and H. Samet. 2011. An incremental Hausdorff distance calculation algorithm. Proc. VLDB 4, 8 (Aug. 2011), 506-517.

[36] S. Nutanong and H. Samet. 2013. Memory-efficient algorithms for spatial network queries. In Proceedings of the 29th IEEE International Conference on Data Engineering. 649-660.

[37] M. Paglione and R. Oaks. 2007. Implementation and metrics for a trajectory prediction validation methodology. In Proceedings of the AIAA GNC Conference and Exhibit. 1-18.

[38] S. Peng and H. Samet. 2015. Analytical queries on road networks: An experimental evaluation of two system architectures. In Proceedings of the 23rd ACM SIGSPATIAL International Conference on Advances in Geographic Information Systems. 
[39] S. Peng and H. Samet. 2018. DOS: A spatial system offering extremely high-throughput road distance computations. In Proceedings of the 26th ACM SIGSPATIAL International Conference on Advances in Geographic Information Systems. 199-208.

[40] S. Peng, J. Sankaranarayanan, and H. Samet. 2016. SPDO: High-throughput road distance computations on spark using distance oracles. In Proceedings of the 32nd IEEE International Conference on Data Engineering. 1239-1250.

[41] M. Prandini, J. Hu, J. Lygeros, and S. Sastry. 2000. A probabilistic approach to aircraft conflict detection. IEEE Trans. Intell. Transport. Syst. 1, 4 (Dec. 2000), 199-220.

[42] M. Prandini, J. Lygeros, A. Nilim, and S. Sastry. 1999. A probabilistic framework for aircraft conflict detection. In Proceedings of the AIAA GNC Conference and Exhibit. 1047-1057.

[43] L. Rabiner. 1990. A tutorial on hidden Markov models and selected applications in speech recognition. In Readings in Speech Recognition. Morgan Kaufmann, San Francisco, CA, 267-296.

[44] H. Samet. 1983. A quadtree medial axis transform.Commun. ACM 26, 9 (Sep. 1983), 680-693.

[45] H. Samet. 1985. Reconstruction of quadtrees from quadtree medial axis transforms.Comput. Vis. Graph. Image Process. 29, 3 (Mar. 1985), 311-328.

[46] H. Samet. 2006. Foundations of Multidimensional and Metric Data Structures. Morgan-Kaufmann, San Francisco, CA.

[47] H. Samet, H. Alborzi, F. Brabec, C. Esperança, G. R. Hjaltason, F. Morgan, and E. Tanin. 2003. Use of the SAND spatial browser for digital government applications. Commun. ACM 46, 1 (Jan. 2003), 63-66.

[48] H. Samet and M. Tamminen. 1986. An improved approach to connected component labeling of images. In Proceedings of the Computer Vision and Pattern Recognition Conference. 312-318.

[49] J. Sankaranarayanan, H. Alborzi, and H. Samet. 2005. Efficient query processing on spatial networks. In Proceedings of the 13th ACM International Symposium on Advances in Geographic Information Systems. 200-209.

[50] J. Sankaranarayanan, H. Alborzi, and H. Samet. 2006. Distance join queries on spatial networks. In Proceedings of the 14th ACM International Symposium on Advances in Geographic Information Systems. 211-218.

[51] J. Sankaranarayanan and H. Samet. 2009. Distance oracles for spatial networks. In Proceedings of the 25th IEEE International Conference on Data Engineering. 652-663.

[52] J. Sankaranarayanan and H. Samet. 2010. Query processing using distance oracles for spatial networks. IEEE Trans. Knowl. Data Eng. 22, 8 (Aug. 2010), 1158-1175.

[53] J. Sankaranarayanan and H. Samet. 2010. Roads belong in databases. IEEE Data Eng. Bull. 33, 2 (Jun. 2010), 4-11.

[54] J. Sankaranarayanan, H. Samet, and H. Alborzi. 2009. Path oracles for spatial networks. Proc. VLDB 2, 1 (Aug. 2009), $1210-1221$.

[55] SESAR. 1999. Single European Sky ATM Research. Retrieved from http://www.eurocontrol.int/dossiers/singleeuropean-sky.

[56] SESAR. 2017. DART. Retrieved from http://dart-research.eu/the-project/.

[57] C. A. Shaffer and H. Samet. 1988. An algorithm to expand regions represented by linear quadtrees. Image Vis. Comput. 6, 3 (Aug. 1988), 162-168.

[58] M. Shewchun, J. Oh, and E. Feron. 1997. Linear matrix inequalities for free flight conflict problems. In Proceedings of the 36th IEEE Conference on Decision and Control. 2417-2422.

[59] M. Soler, A. Olivares, E. Staffetti, and J. Cegarra. 2011. Multi-phase optimal control applied to 4D business trajectory strategic planning in air traffic management. In Proceedings of the 1st International Conference on ATACCS. 68-78.

[60] E. Tanin, A. Harwood, and H. Samet. 2005. A distributed quadtree index for peer-to-peer settings. In Proceedings of the 21st IEEE International Conference on Data Engineering. 254-255.

[61] A. Valenzuela and D. Rivas. 2009. Conflict detection and resolution in converging air traffic. In Proceedings of the 9th AIAA ATIO Conference. 1-13.

[62] A. Vink, S. Kauppinen, J. Beers, and K. Jong. 1997. Medium-term conflict detection in EATCHIP phase III. In Proceedings of the 16th DASC AIAA/IEEE Digital Avionics Systems Conference. 45-52.

[63] A. Viterbi. 1967. Error bounds for convolutional codes and an asymptotically optimum decoding algorithm. IEEE Trans. Inf. Theory 13, 2 (Apr. 1967), 260-269.

[64] L. Yang and J. Kuchar. 1998. Using intent information in probabilistic conflict analysis. In Proceedings of the AIAA GNC Conference and Exhibit. 797-806.

[65] Y. Yang, K. Cai, and M. Prandini. 2017. Fast algorithm based on computational geometry for probabilistic aircraft conflict detection. In Proceedings of the 2017 International Conference on Robotics and Artificial Intelligence. 66-70.

[66] K. Zeghal and E. Hoffman. 1999. Design of cockpit displays for limited delegation of separation assurance. In Proceedings of the 18th Digital Avionics Systems Conference. 1-8.

Received December 2018; revised April 2019; accepted April 2019 